This item was submitted to Loughborough's Research Repository by the author.

Items in Figshare are protected by copyright, with all rights reserved, unless otherwise indicated.

\title{
Thermodynamics of entropy-driven phase transformations
}

PLEASE CITE THE PUBLISHED VERSION

PUBLISHER

(C) American Physical Society

LICENCE

CC BY-NC-ND 4.0

\section{REPOSITORY RECORD}

Radosz, A., K. Ostasiewicz, P. Magnuszewski, J. Damczyk, L. Radosiński, F.V. Kusmartsev, and J.H. Samson. 2019. "Thermodynamics of Entropy-driven Phase Transformations". figshare. https://hdl.handle.net/2134/1300. 


\title{
Thermodynamics of entropy-driven phase transformations
}

\author{
A. Radosz, ${ }^{1}$ K. Ostasiewicz, ${ }^{1}$ P. Magnuszewski, ${ }^{1}$ J. Damczyk, ${ }^{1}$ Ł. Radosiński, ${ }^{1}$ F. V. Kusmartsev, ${ }^{2}$ J. H. Samson, ${ }^{2}$ \\ A. C. Mituś, ${ }^{1}$ and G. Pawlik ${ }^{1}$ \\ ${ }^{1}$ Institute of Physics, Wroctaw University of Technology, 50-370 Wroctaw, Poland \\ ${ }^{2}$ Department of Physics, Loughborough University, Loughborough LE11 3TU, United Kingdom
}

(Received 10 March 2005; revised manuscript received 27 September 2005; published 24 February 2006)

\begin{abstract}
Thermodynamic properties of one-dimensional lattice models exhibiting entropy-driven phase transformations are discussed in quantum and classical regimes. Motivated by the multistability of compounds exhibiting photoinduced phase transitions, we consider systems with asymmetric, double, and triple well on-site potential. One finds that among a variety of regimes, quantum versus classical, discrete versus continuum, a key feature is asymmetry distinguished as a "shift" type and "shape" type in limiting cases. The behavior of the specific heat indicates one phase transformation in a "shift" type and a sequence of two phase transformations in "shape"-type systems. Future analysis in higher dimensions should allow us to identify which of these entropydriven phase transformations would evolve into phase transitions of the first order.
\end{abstract}

DOI: 10.1103/PhysRevE.73.026127

PACS number(s): 05.70.-a, 64.60.-i, 63.70.+h

\section{INTRODUCTION}

One-dimensional (1D) or quasi-one-dimensional strongly nonlinear systems exhibit a variety of interesting phenomena. Lattice systems with degenerate vacuum, supporting topological excitations are applied to hydrogen-bonded complexes: kinks are responsible for the proton transport through the chains of hydrogen bonds [1,2]. Localized excitations such as kinks or solitons also play an important role in dynamical phenomena in biological systems $[3,4]$. In the thermodynamic context, the coexistence of localized excitations (kinks) and extended excitations (phonons) manifests itself via a characteristic hump developed in the specific heat $[5,6]$. Two-dimensional and three-dimensional versions of systems with degenerate vacua exhibit phase transitions of second order-in fact, it is the simplest manifestation of phenomena of spontaneously broken (discrete) symmetry. It was shown by Morris and Gooding [7] that systems with nondegenerate vacua exhibit first-order phase transitions and that analysis of 1D systems provides some insight into the properties of systems of higher dimensions.

Recent discoveries and progress in understanding of the phenomena commonly referred to as photoinduced phase transitions [8-14] have turned attention to nonlinear systems with nondegenerate vacua. The remarkable properties of that class of systems are their strongly anisotropic, nearly onedimensional character, first-order phase transition, which might be light-induced, and multistability. Multistability is of particular character: two nondegenerate, usually distinct phases, a stable one and a metastable one, e.g., ionic and neutral in charge-transfer systems [8], may be distinguished. Among a variety of phenomena and materials exhibiting photoinduced phase conversion, the most popular are chargetransfer and spin-conversion compounds. In charge-transfer systems, the ground state is an ionic dimerized phase and the neutral phase corresponds to a metastable state. That class of systems reveals a first-order phase transition from a lowtemperature ionic phase to a high-temperature neutral phase. In spin-conversion or spin-crossover systems, the low spin state corresponds to a ground state and the high spin state corresponds to a metastable state [14]. A first-order phase transition takes place between these two states. Dynamical phenomena of photoinduced phase transformations depend strongly on electron-phonon coupling and it seems that phase conversion, which also might be light-induced, is a transition from a stable to a metastable state. The lower, ground state is of smaller entropy than the higher, metastable state. Phase transformation in these highly anisotropic, nearly onedimensional systems is an example of entropy-driven conversion.

In different attempts at describing the complexity of photo-induced phase conversions, two types of approaches might be identified. In the Peierls-Hubbard-type approach $[8,13,14]$, electronic excitations are regarded as delocalized, belonging to two and four narrow bands in neutral and ionic phases, respectively. In the other class of approaches, attempting to describe some dynamic electron-phonon features [9] and thermodynamic properties of charge-transfer and spin-crossover [14] photoinduced phase-transition systems, two or three states of localized electrons coupled to phonon system are taken into account. That approach may lead to a lattice, local, asymmetric double-well potential $[9,14]$ or triple-well potential [15]. Such a multiple-well, on-site potential corresponds to an electronic ground state. We discuss here the properties of such a minimal lattice model with local asymmetric double-well or triple-well potential.

Our purpose is to give a thorough discussion of the thermodynamic properties of one-dimensional lattice models of systems developing entropy-driven phase transformation. In such systems one can observe an interesting thermodynamic behavior clearly manifested in the specific heat. Examination of the analytic form of thermodynamic functions in the classical limit provides a detailed description of the phase transformations occurring in that class of systems. Going beyond the classical limit, one can describe the role of quantum corrections: though quantum corrections influence essentially specific heat as a function of temperature in a quantum regime, the qualitative character of phase conversion is preserved. Discreteness of the systems is also taken into account: entropy-driven phase transformation apparent in the 
continuum limit of large asymmetry systems, gradually damped with growing discreteness, turns out eventually to be smeared out. We apply here a particular form of an on-site double-well potential as composed of two Morse potentials - a double Morse potential-that belongs to the class of (quasi)exactly solvable quantum-mechanical models. It enables us to give an analytical formula in a part of our qualitative discussion.

Our paper is organized in the following way. In the next section, we introduce the model and formulate the problem. In Sec. III, the partition function within the FeynmanKleinert approach is briefly considered. The one-dimensional character of the problem converts the question of finding the partition function and thermodynamic functions into the problem of solving some eigenvalue integral or differential (pseudo)Schrödinger equation. The method of taking into account quantum effects is described and a semiclassical approach is applied. In Sec. IV, the thermodynamics of the model system with a double-well potential is investigated in the classical limit; the quantum regime and effects of discreteness are discussed in Sec. V. In Sec. VI, the properties of systems with a triple-well potential are considered. Discussion and final remarks are given in Sec. VII. Appendixes A and B contain technical details: a description of the evaluation of the partition function in the quantum regime (Appendix A) and a semiclassical approach for the quantummechanical eigenvalue problem in a case of a multiple-well potential (Appendix B).

\section{FORMULATION OF THE PROBLEM}

We shall consider a one-dimensional (1D), onecomponent system of harmonically coupled atoms with a local asymmetric double-well or triple-well potential. The Hamiltonian of such a system will be written in the form [5]

$$
H=\sum_{n} \frac{m \omega_{0}^{2}}{\alpha^{2}}\left(\frac{1}{2} \dot{x}_{n}^{2}+\frac{1}{2} k\left(x_{n+1}-x_{n}\right)^{2}+V\left(x_{n}\right)\right),
$$

where $\alpha$ denotes the characteristic inverse distance, $\omega_{0}$ is a characteristic frequency, and $x_{n}$ is the dimensionless coordinate at the $n$th lattice site. $\dot{x}_{n}$ denotes (dimensionless) time derivative. The double-well on-site potential is chosen as an asymmetric double Morse potential,

$$
\begin{aligned}
V_{\mathrm{DM}}\left(x_{n}\right)= & V_{1}\left(e^{-2 \alpha_{1}\left(x_{n}+q_{0}\right)}-2 e^{-\alpha_{1}\left(x_{n}+q_{0}\right)}\right)+V_{2}\left(e^{2 \alpha_{2}\left(x_{n}-q_{0}\right)}\right. \\
& \left.-2 e^{\alpha_{2}\left(x_{n}-q_{0}\right)}\right) .
\end{aligned}
$$

The triple-well potential, introduced in Sec. V, is composed of the symmetric potential (2) and the central Pöschl-Teller potential. The double Morse potential, composed of two Morse single-well potentials, has a richer structure than other bistable potentials, e.g., " $\phi^{4}$," and was found to be a suitable tool for modeling realistic situations like the hydrogen bond. Nevertheless, most of our results and predictions are independent of the choice of a particular form of an on-site potential.

Thermodynamic properties are studied by using the partition function

$$
Z=\operatorname{Tr} e^{-\beta H} .
$$

That partition function treated rigorously takes the form of the functional integral,

$$
\begin{aligned}
Z=\operatorname{Tr} e^{-\beta H} & =\int \prod_{n} d x_{n}^{\prime}\left\langle\bar{x}^{\prime}\left|e^{-\beta H}\right| \bar{x}^{\prime}\right\rangle \\
& =\int \prod_{n} D\left[x_{n}(\tau)\right] \exp \left(-\frac{1}{\hbar} A[\bar{x}(\tau)]\right),
\end{aligned}
$$

where the action $A$ is an integral over imaginary time (see Appendix A). The classical limit, usually regarded as a hightemperature approach, arises when noncommutating coordinate and momentum operators are substituted by real valued numbers. Then the partition function $Z$ is by definition expressed as an integral over all momentum and coordinates,

$$
\begin{gathered}
Z_{\mathrm{cl}}=\left[\frac{\gamma}{2 \pi}\right]^{N} \int d p^{N} d x^{N} \exp \left\{-\beta \gamma \sum_{n}\left[\frac{p_{n}^{2}}{2}+V\left(x_{n}-x_{n-1}\right)\right]\right\} \\
V\left(x_{n}, x_{n-1}\right)=\frac{1}{2} k\left(x_{n}-x_{n-1}\right)^{2}+V\left(x_{n}\right),
\end{gathered}
$$

where $1 / \beta$ is measured in $\hbar \omega_{0}$ units, and $\gamma \equiv m \omega_{0} / \hbar \alpha^{2}$.

The problem of finding a partition function in a 1D system might be converted into an integral operator eigenvalue problem in that limit; in the continuum limit, that eigenvalue problem takes the form of a differential, pseudo-Schrödinger equation. In the framework of a quantum approach to 1D nonlinear systems (1), rather limited results have been produced so far. As was noticed by Gütlich et al. [16] and Boukheddaden et al. $[10,11]$, in the case of systems displaying spin-crossover transformations, quantum aspects may be essential even at room temperature. Hence we are considering here an approach that goes beyond the classical approximation, and quantum features are taken into account from the very beginning: the partition function is treated as a functional integral, and a classical approximation arises in a natural way as a high-temperature limit.

\section{PARTITION FUNCTION: QUANTUM APPROACH AND CLASSICAL LIMIT}

In order to make an approximate evaluation of the partition function in the framework of a quantum approach, we invoke a Feynman-Kleinert approximation. Originally, the Feynman-Kleinert method was designed for zerodimensional, one-particle problems. The key element of that approximation is a procedure of averaging over quantum fluctuations performed in a variational way leading to a pseudoclassical partition function. Applying that approach to the 1D system (see Appendix A), one obtains a semiclassical form of the partition function with some effective, temperature-dependent potential $[17,18]$ [see Eq. (7)],

$$
Z_{\mathrm{scl}}=\left[\frac{\gamma}{2 \pi \beta}\right]^{N / 2} \int_{n=1}^{N} d x_{n} \exp \left\{-\beta \gamma \tilde{V}^{\mathrm{eff}}\left(\left\{x_{n}\right\}\right)\right\} .
$$

An effective potential is given by (see Appendix A) 


$$
\tilde{V}^{\mathrm{eff}}=\sum_{n} \frac{1}{2} k\left(x_{n}-x_{n-1}\right)^{2}+\frac{1}{2} M \sum_{q}\left[\left(\omega_{q}^{2}-\widetilde{\omega}_{q}^{2}\right) a_{q}^{2}\right]+F_{0}+V_{\mathrm{anh}}^{\mathrm{eff}},
$$

where

$$
\begin{gathered}
\omega_{q}^{2}=4 k \sin ^{2}\left[\frac{\pi}{N} q\right], \\
F_{0}=-k_{B} T \sum_{q} \ln \left[\frac{\frac{\hbar \widetilde{\omega}_{q}}{2 k_{B} T}}{\sinh \left(\frac{\hbar \widetilde{\omega}_{q}}{2 k_{B} T}\right)}\right], \\
a_{q}^{2}=\frac{k_{B} T}{M \widetilde{\omega}_{q}^{2}}\left[\frac{\hbar \widetilde{\omega}_{q}}{2 k_{B} T} \operatorname{coth} \frac{\hbar \widetilde{\omega}_{q}}{2 k_{B} T}-1\right] .
\end{gathered}
$$

Trial frequencies are taken from the minimization condition,

$$
\frac{\partial V_{\mathrm{anh}}^{\mathrm{eff}}}{\partial \widetilde{\omega}_{q}^{2}}=0 \Rightarrow \omega_{q}^{2}-\widetilde{\omega}_{q}^{2}=\frac{2}{M} \frac{\partial V_{\mathrm{anh}}^{\mathrm{eff}}}{\partial a_{q}^{2}},
$$

and an effective anharmonic potential is, in the case of a double-Morse (DM) potential and in the limit of $N \rightarrow \infty$, given as

$$
\begin{gathered}
V_{\mathrm{DM}}^{\mathrm{eff}}\left(\left\{x_{n}\right\}\right)=\sum_{n}\left[V_{1}\left(e^{-2 \alpha_{1}\left(x_{n}+q_{0}\right)} e^{2 \alpha_{1}^{2} D}-2 e^{-\alpha_{1}\left(x_{n}+q_{0}\right)} e^{(1 / 2) \alpha_{1}^{2} D}\right)\right. \\
\left.+V_{2}\left(e^{2 \alpha_{2}\left(x_{n}-q_{0}\right)} e^{2 \alpha_{2}^{2} D}-2 e^{\alpha_{2}\left(x_{n}-q_{0}\right)} e^{(1 / 2) \alpha_{2}^{2} D}\right)\right] \\
D=\frac{1}{N} \sum_{q} a_{q}^{2}
\end{gathered}
$$

The semiclassical form (6) of the partition function suggests an implementation of an approach applied in the classical limit, namely the transfer-matrix method; then the thermodynamic properties of such a system may be investigated by means of the lowest eigenvalue of an appropriate integral/ differential equation. In the classical limit, the transfermatrix method yields an eigenvalue problem with an on-site potential, and the corresponding quantum-mechanical problem allows for a qualitative discussion. Feynman-Kleinert treatment provides us with an on-site potential substituted by an effective, temperature-dependent one and a qualitative discussion appears to be very limited. The free energy of the system in the continuum limit is determined by the lowest eigenvalue of the Schrödinger-like equation,

$$
\begin{aligned}
& \left\{-\frac{d^{2}}{d u^{2}}+2 \beta^{2} k \gamma^{2} V_{\mathrm{anh}}^{\mathrm{eff}}(u)\right\} \Psi_{n}(u) \\
& =2 \beta^{2} k \gamma\left[E_{n}+\frac{1}{2 \beta} \ln \frac{2 \pi}{\beta k \gamma}\right] \Psi_{n}(u) .
\end{aligned}
$$

Numerical methods are invoked here (see the next section) to study the properties of that continuum limit. Before discussing the thermodynamics determined by partition function (6), let us make some general remarks. First, let us no-

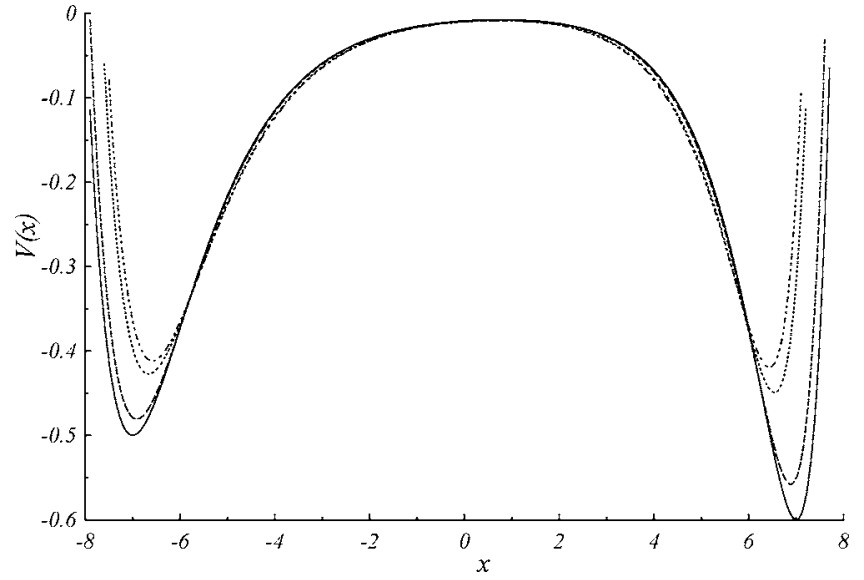

FIG. 1. The shape of the effective potential for values of parameters: $V_{1}=0.5, V_{2}=0.6, \alpha_{1}=0.7, \alpha_{2}=0.95, q_{0}=7, k=1$, and $\gamma=1$, for three values of temperatures: $T=0.001$ (dash-dot-dotted line); $T$ $=0.1$ (dotted line) $T=1$ (dashed line $)$-compared to the classical potential (solid line).

tice that the form of the effective on-site potential gradually evolves with temperature toward the original one in the hightemperature limit (see Fig. 1). The procedure of averaging over quantum fluctuations results in a smoother shape of the effective potential than the original, i.e., lowering the potential barrier and level of asymmetry. Second, one can find a variety of regimes and limits in a class of systems with an asymmetric double-well on-site potential. One can distinguish classical and quantum regimes and continuum and discrete limits; also asymmetry might be, as we will see, quantified. Three characteristic energy parameters might be identified in that context: thermal fluctuations, $k_{B} T$, quantum fluctuations, i.e., zero-point energy, $\hbar \omega_{0}$, and the height of the energy barrier of the on-site potential. Interplay among these quantities identifies the expected corresponding regimes. The classical limit is reached when the characteristic energy, $k_{B} T$, is large compared to the energy of quantum fluctuations, $\hbar \omega_{0}$. One can expect that interesting phenomena will be encountered in the range of energies below the barrier of the on-site potential. The ratio of the quantity $m \omega_{0}^{2} / \alpha^{2}$, proportional to the height of the barrier of the on-site potential, and the energy of quantum fluctuations, $\hbar \omega_{0}$, denoted as $\gamma$, may be referred to as a parameter measuring the influence of quantum effects. A large value of $\gamma$ corresponds to the systems where quantum effects are limited to the lowtemperature range and may be regarded as small quantum corrections. A small value of $\gamma$ corresponds to the systems where interesting nonlinear effects are observed within the quantum regime. An attempt to quantify a level of asymmetry is made and systems with small and large asymmetry are identified. Such a division could not be rigorous, but we can distinguish these two cases on the basis of a criterion of "crossing" or "noncrossing" "single-well" levels (see below). It results from the fact that the double-well on-site potential as composed of the two wells is characterized by the shape of the two wells and their mutual arrangement. The conclusion for a triple-well potential is straightforward.

One can also distinguish between discrete and continuum limits. As a strong coupling between atoms corresponds to 
the continuum limit, a suitable parameter, $k$, describes the ratio of the harmonic interaction energy and the height of the barrier of the on-site potential. Hence, classical and quantum, continuum and discrete regimes in small and large asymmetry systems may be considered as particular limits. However, as we shall see, thermodynamic properties appear to depend effectively on the level of asymmetry of the on-site potential. Thermodynamic characteristics related to classical versus quantum and continuum versus discrete limits, rather easily predicted, are not leading (in thermodynamics) to qualitatively new phenomena. Therefore, we shall consider the situation of a triple-well potential, in the classical limit, for the case of two symmetric side wells on either side of a central well (deeper or shallower).

\section{CLASSICAL THERMODYNAMICS IN THE CONTINUUM LIMIT $\gamma \gg 1, k>1$ : SYSTEMS WITH AN ASYMMETRIC DOUBLE-WELL POTENTIAL}

Classical thermodynamics is generally regarded as a hightemperature limit, where the noncommutative character of the variables-operators is ignored. In the framework of the Feynman-Kleinert approach it arises naturally, as the energy of any quantum-mechanical excitation is much smaller than the classical energy, $k_{B} T$. Then one finds

$$
\lim _{\beta \rightarrow 0} \frac{1}{\gamma \beta \omega_{q}^{2}\left(\left\{x_{n}\right\}\right)}\left[\frac{\beta \omega_{q}\left(\left\{x_{n}\right\}\right)}{2} \operatorname{coth} \frac{\gamma \beta \omega_{q}\left(\left\{x_{n}\right\}\right)}{2}-1\right]=0,
$$

i.e., $a^{2}=0$; that fully reproduces the classical approach: quantum fluctuations disappear. In this case, quantum effects are limited to low temperatures, $k_{B} T \ll\left[V_{\max }-V_{\min }\right]$ (see Fig. 14 in Appendix B). The high-temperature limit (11) restores for the effective potential in the quantum partition function (4) its original form (2). Then partition function $Z$ takes the form

$$
Z=\left[\frac{\gamma}{2 \pi \beta}\right]^{N / 2} \int \prod_{n=1}^{N} d x_{n} \exp \left\{-\beta \gamma V\left(x_{n}, x_{n-1}\right)\right\},
$$

which actually is a classical partition function (5) averaged over momentum $p$,

$$
Z_{c l}=\left[\frac{\gamma}{2 \pi \beta}\right]^{N / 2} \sum_{n} e^{-\beta N E_{n}(\beta)} .
$$

The configurational part of the partition function is expressed via eigenvalues of an appropriate integral operator $K$ (see, e.g., Morris and Gooding [7]),

$$
\int K(x, y) \Psi_{n}(y) d y=\varepsilon_{n} \Psi_{n}(x), \quad \varepsilon_{n}=e^{-\beta E_{n}}
$$

defined as

$$
K(x, y)=\exp \left\{-\beta\left[\frac{1}{2} \gamma V(x)+\frac{1}{2} k \gamma(x-y)^{2}+\frac{1}{2} \gamma V(y)\right]\right\} .
$$

In the continuum limit, $k>1$, this integral eigenvalue problem is converted into a differential, Schrödingerequation-like problem,

$$
\begin{gathered}
e^{-(1 / 2) \beta \gamma V(x)} e^{(1 / 2) \ln (2 \pi / \beta k \gamma)} e^{(1 / 2 \beta k \gamma)\left(d^{2} / d x^{2}\right)} e^{-(1 / 2) \beta \gamma V(x)} \Psi_{n}(x) \\
=e^{-\beta E_{n}} \Psi_{n}(x), \\
{\left[-\frac{1}{2 m^{*}} \frac{d^{2}}{d x^{2}}+V(x)\right] \Psi_{n}(x)=\left[E_{n}+\frac{1}{2 \beta} \ln \frac{2 \pi}{\beta k \gamma}\right] \Psi_{n}(x)} \\
m^{*} \equiv \beta^{2} k \gamma^{2} .
\end{gathered}
$$

The ground-state eigenvalue of that equation is simply related to the free energy of the system,

$$
\begin{aligned}
F & =-\lim _{N \rightarrow \infty} \frac{1}{N \beta} \ln Z_{\mathrm{cl}} \\
& =-\frac{1}{2 \beta} \ln \left[\frac{\gamma}{2 \pi \beta}\right]+E_{0}+\lim _{N \rightarrow \infty} \ln \left[1+\sum_{n \geqslant 1} e^{-\beta N \Delta_{0 n}}\right] \\
& =-\frac{1}{2 \beta} \ln \left[\frac{\gamma}{2 \pi \beta}\right]+E_{0},
\end{aligned}
$$

and mean energy, entropy, and specific heat are expressed via the ground-state eigenvalue and its first- and second-order derivatives,

$$
U \equiv\langle H\rangle=-\frac{1}{Z_{c l}} \frac{\partial}{\partial \beta} Z_{\mathrm{cl}}=\frac{\partial}{\partial \beta}(\beta F)=\frac{1}{2 \beta}+E_{0}+\beta \frac{\partial E_{0}}{\partial \beta},
$$

$$
\begin{gathered}
S=\frac{U-F}{T}=-\frac{\partial F}{\partial T}, \\
C_{V}=\frac{\partial}{\partial T} U=\frac{1}{2}-T \frac{\partial^{2} E_{0}}{\partial T^{2}} .
\end{gathered}
$$

The probability distribution function (PDF) equals the squared ground-state eigenfunction and is interpreted as a probability density of finding a certain value of the on-site potential for the particle's coordinate [19].

The effective mass in the pseudo-Schrödinger equation (10) is proportional to the inverse square temperature. The high-temperature limit corresponds then to a "light particle" and ground state placed above the barrier of the on-site potential; in this limit, the PDF turns out to be centrally centered, nearly independent of the form of an on-site potential. The low-temperature limit corresponds to a "heavy particle" and ground state placed well below the edge of the potential barrier - the properties of the solution of Eq. (10) are analyzed in Appendix B by using the semiclassical approach called RTCT (real trajectories in complex time) [20].

That method provides us with a description of temperature evolution of the "double-well" ground state of the system $E_{0}$ as related to temperature evolution of "single-well" ground states $E_{0}^{1}$ and $E_{0}^{2}$. These "single-well" states correspond to the classical free energy (configuration part) of two "single-well" phases: harmonically coupled oscillators with a left and right Morse well, as an on-site potential. The evolution of a pure state, described here analytically (Appendix B), 


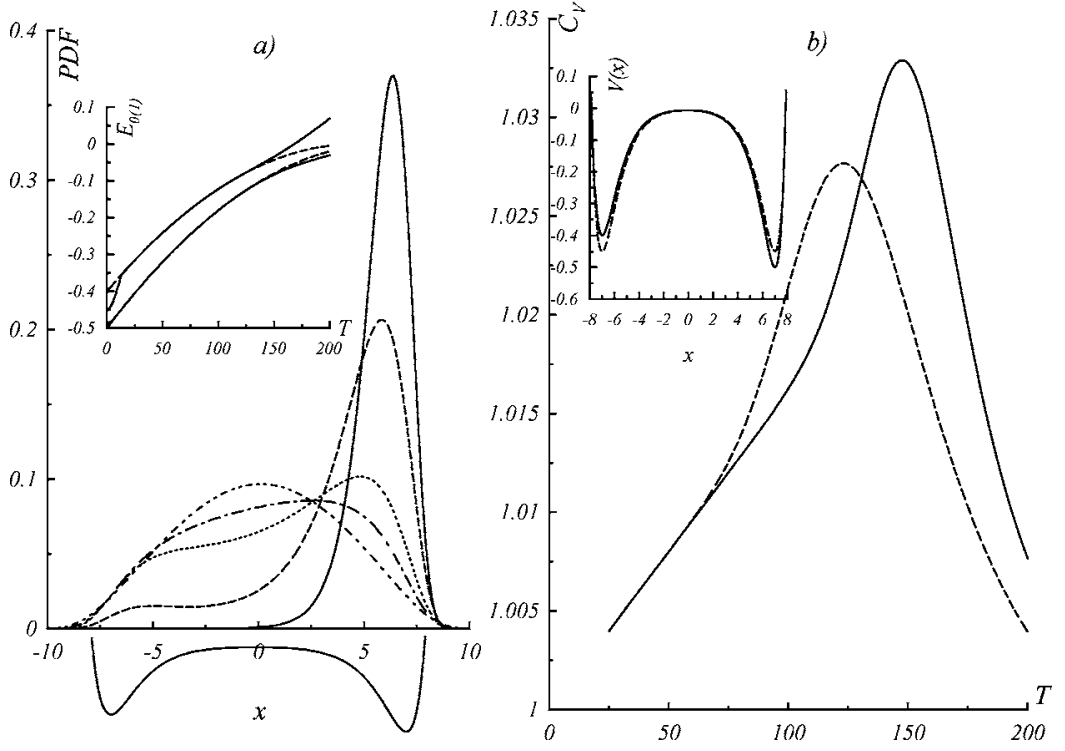

$$
E_{0}^{i}=-\frac{T^{2}}{k} \frac{\alpha_{i}^{2}}{2 \gamma^{2}}\left[\frac{1}{2}-\sqrt{\frac{2 \gamma^{2} k V_{i}}{\alpha_{i}^{2} T^{2}}}\right]^{2},
$$

depends on the shape of the potential well: evolution in a narrower well is faster than the evolution in a broader well. That leads to two different types of asymmetry associated with two different scenarios of temperature evolution.

\section{A. Small asymmetry—"Shift" type}

Let us begin with an on-site potential composed of the two identical (face-to-face) Morse wells shifted against each other, $\alpha_{1}=\alpha_{2}, V_{1} \neq V_{2}$. Single-well states are evolving in the same way and the distance between them, $E_{0}^{2}-E_{0}^{1}\left(V_{1}>V_{2}\right)$, is (nearly) temperature-independent [see Fig. 2(a)]; the double-well ground state corresponds to the lower of the single-well states. The temperature behavior of the groundstate eigenfuction and PDF is rather smooth: at low temperatures, the PDF is centered in the lower well, gradually leaking to the higher well [Fig. 2(a)]. Such behavior is accompanied by the emergence of the hump in the specific heat [Fig. 2(b)]. That hump, characteristic of the symmetric on-site potential [dashed line in Fig. 2(b)], was interpreted as responsible for the domain-wall (kinks) movement. More generally, the scenario with a smooth hump in a specific heat and a smooth change of other quantities takes place in systems with a (nearly) uniform evolution of single-well states. We will refer to such systems as a small asymmetry, or "shift" type asymmetry: the difference $E_{0}^{2}-E_{0}^{1}$ only depends weakly on the temperature. Namely, in systems of small or shift type asymmetry, single-well ground states do not cross each other.

\section{B. Large asymmetry - "Shape" type}

One can define then the systems of large asymmetry"shape" type-as those in which the single-well ground energy distance $E_{0}^{2}-E_{0}^{1}$ varies with temperature, and vanishes at some particular temperature. Namely, if the single-well ground states cross each other, the system is referred to as a large, or shape-type asymmetry. Such a level crossing would happen in the case of an on-site potential composed of two wells of different shapes, $\alpha_{1} \neq \alpha_{2}$, when the lower well becomes narrower than the shallower one. That is a necessary condition; a satisfactory one is that single-well level crossing has to take place before they would reach the edge of the potential barrier. When the single-well states are getting closer, $\left|E_{0}^{1}-E_{0}^{2}\right|<E_{1}^{1}-E_{0}^{1}$, double-well states are forming characteristic pairs. A pair corresponding to the ground state is given as (see Appendix B)

$$
\begin{gathered}
E_{0}^{\mp}=\frac{1}{2}\left[\left(E_{0}^{1}-E_{0}^{2}\right) \mp \sqrt{\left(E_{0}^{1}-E_{0}^{2}\right)^{2}+b^{2}\left(E_{0}\right) \nu_{1} \nu_{2}}\right], \\
\nu_{i}=\left[\left.\frac{\partial W_{i}}{\partial E}\right|_{E=E_{i}^{0}}\right]^{-1} .
\end{gathered}
$$

Then the intersection of the ground states $E_{0}^{1}$ and $E_{0}^{2}$ happens at temperature $T^{*}$,

$$
T^{*}=\frac{2 \gamma \sqrt{2 k}}{\alpha_{2}-\alpha_{1}}\left[\sqrt{V_{2}}-\sqrt{V_{1}}\right] .
$$

That effect is not directly observed, but avoided level crossing happens [Fig. 3(a)], hidden behind a smooth change of $E_{0}^{-}(T)$ (Fig. 3). However, avoided level crossing is expected to result in a particular sort of phase transformation and should have quite dramatic consequences for other characteristics of the system. Indeed, the PDF reveals a "rapid jump" between the wells (see Fig. 3). Such a coherent transition between two single-well phases is accompanied by a high and narrow peak in the specific heat, Fig. 3(b). The analytic form of the specific heat in the vicinity of the intersection, $\delta E_{0} \equiv E_{2}^{0}-E_{1}^{0}=0$, found from Eq. (23), 


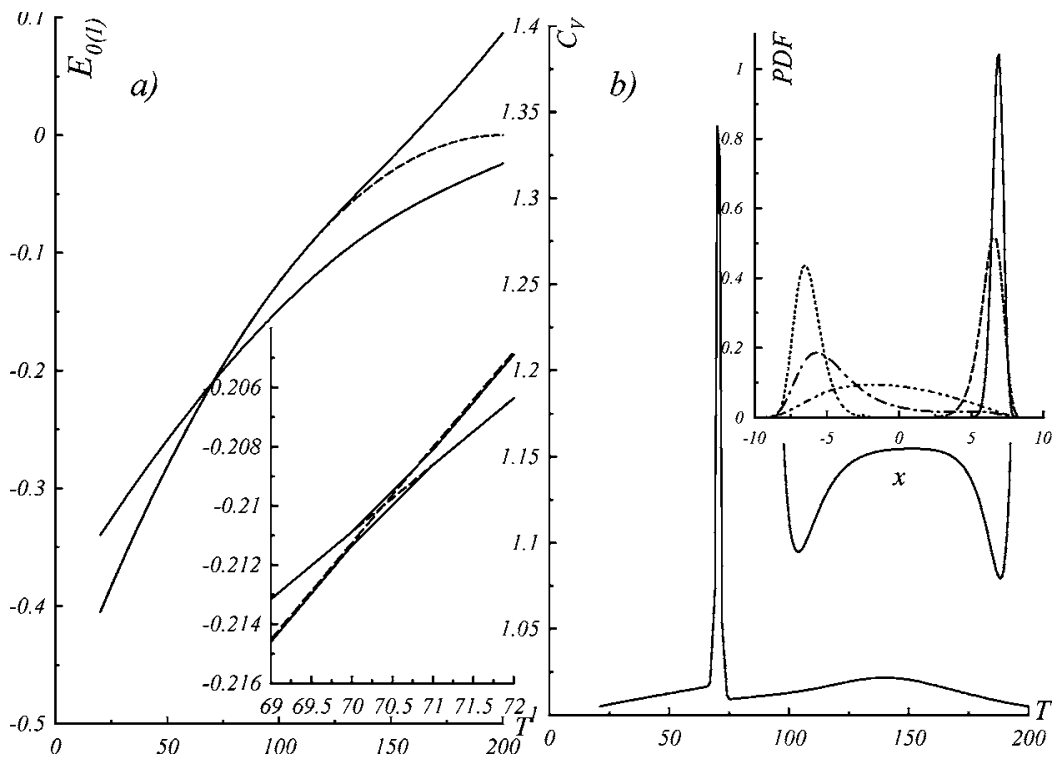

FIG. 3. (a) Avoided level crossing for large asymmetry (values of parameters: $V_{1}=0.4, V_{2}$ $\left.=0.5, \alpha_{1}=0.7, \alpha_{2}=1, q_{0}=7, k=100, \gamma=10\right)$ - $\mathrm{two}$ lowest double-well levels $E_{0}^{\mp}$ (solid lines) and single-well ground levels $E_{1}^{0}, E_{2}^{0}$ (dashed lines) are shown; avoided crossing enlarged in the inset. (b) Specific heat for the same parameters with PDF (inset) for temperatures $T=25$ (solid), $T$ $=70$ (dash), $T=71$ (dot), $T=150$ (dash-dot), $T$ $=300$ (dash-dot-dot).

$$
C_{V} \propto \frac{1}{\sqrt{\left(\delta E_{0}\right)^{2}+b^{2}\left(E_{0}\right) \nu_{1} \nu_{2}}},
$$

indicates nearly singular behavior for a large energy barrier: the height of this peak is proportional to the exponent of the area under the barrier between wells. The two-peak structure of the specific heat in the case of large asymmetry of a particular type reflects two phase transformations.

The first of these transformations, at lower temperature, is sharp, an entropy-driven change from a stable state to a metastable state; the other transformation, at higher temperature, is smoother, corresponding to a change from a metastable state to a high-temperature state. Such a sequence of two phase transformations is reflected in an average "position": indeed, an average position, defined as $\langle x\rangle$ $=\int x d x\left|\Psi_{0}(x)\right|^{2}$, displays an unusual behavior (Fig. 4), corresponding to the above description. It should be emphasized that a smooth temperature dependence in the hightemperature range, drift to a metastable state, and a sharp, "jumplike" change to a stable state at lower temperatures are

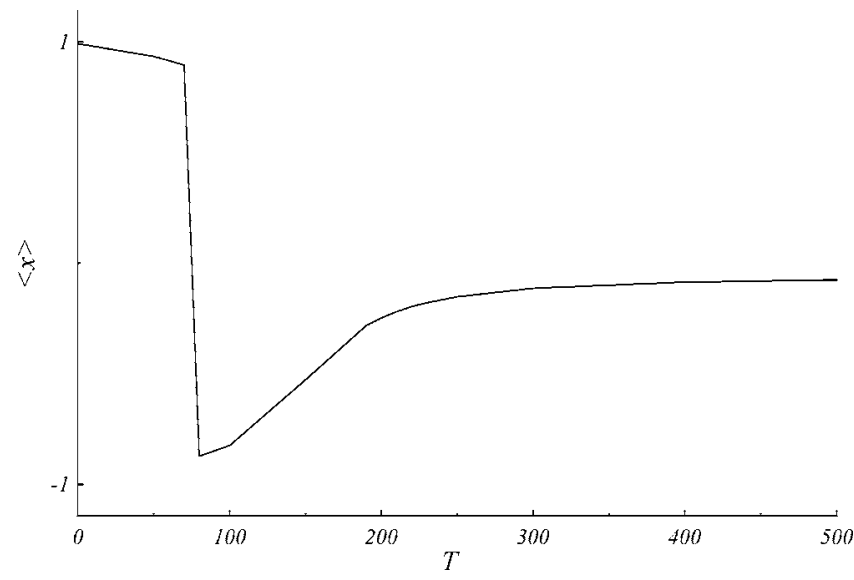

FIG. 4. Average "position" for large asymmetry (values of parameters: $\left.V_{1}=0.4, V_{2}=0.5, \alpha_{1}=0.7, \alpha_{2}=1, q_{0}=7, k=100, \gamma=10\right)$. characteristic of large-asymmetry, strongly coupled (continuum limit) systems.

A dramatic jump in the PDF, accompanied by nearly singular behavior of specific heat, is only weakly manifested in lower-order thermodynamic functions, mean energy, and entropy. In Figs. 5(a) and 5(b), one can see how these functions change, from the form characteristic of the narrower well to the form characteristic for the wider well (results corresponding to pure phases are plotted by dashed lines).

\section{QUANTUM EFFECTS AND EFFECTS OF DISCRETENESS}

\section{A. Quantum regime in the continuum limit: $\gamma \approx 1, k>1$}

Quantum fluctuations enter the picture when the thermal energy is comparable with zero-point fluctuations energy: in the Feynman-Kleinert approach, quantum fluctuations measured by parameter $a^{2}$ are no longer ignored. In the continuum limit, thermodynamic properties are studied by means of the eigenvalues and eigenfunctions of the pseudoSchrödinger Eqs. (10). In the range of large values of parameter $\gamma(\gamma \gg 1$, the classical limit), the effective potential, evolving with temperature, takes its original form (classical limit) well before the interesting effects in specific heat, a hump or two-peak structure, are developed. Specific heat exhibits then a usual behavior: it starts from zero, reaches the high-temperature value, and only then do interesting effects (one or two peaks) appear (in Figs. 6 and 7, see curves $\gamma$ $=10)$.

In that range, quantum fluctuations may be regarded as small corrections. Quantum effects become more important when the values of parameter $\gamma$ become smaller and quantum fluctuations modify significantly the on-site potential within the range of phase transformation. It was stressed earlier [21] that hydrogen-bonded and spin-conversion systems may manifest their quantum character, even at room temperatures, via affecting the shape of the hump in the specific heat. Investigating the systems of small asymmetry, one finds that a decreasing value of $\gamma$ corresponds to an extension of the 


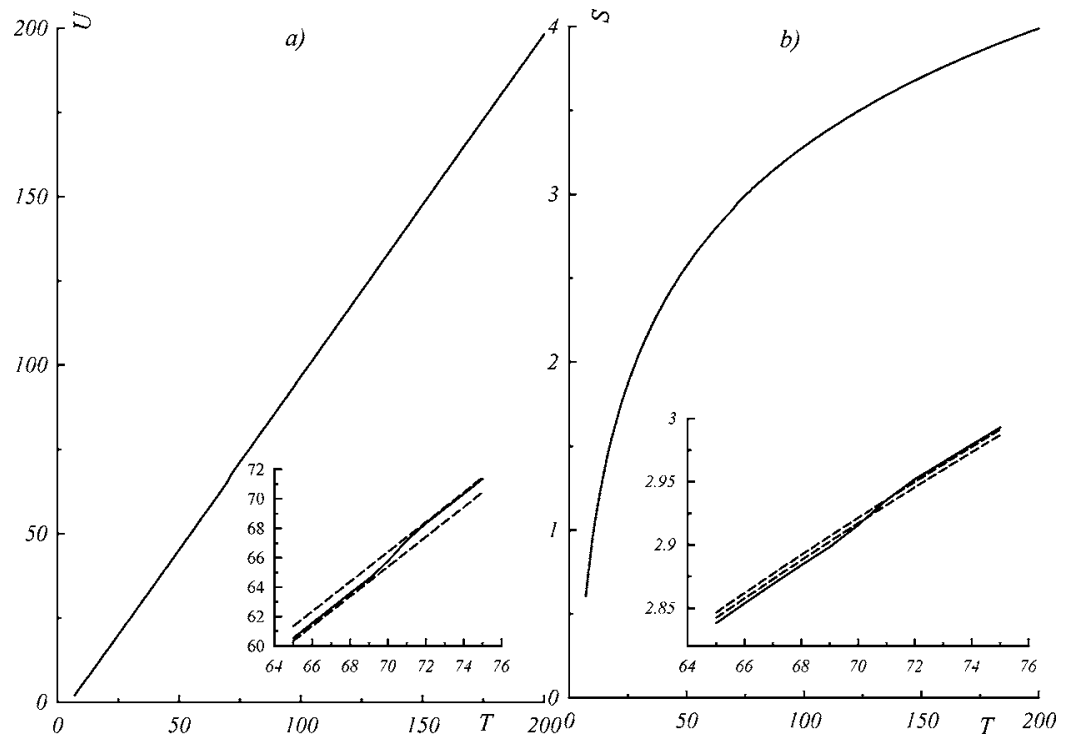

FIG. 5. (a) Mean energy and (b) entropy for parameters: $V_{1}=0.4, V_{2}=0.4, \alpha_{1}=0.7, \alpha_{2}=1, q_{0}$ $=7, k=100, \gamma=10$, with enlarged regions of steps in these functions (insets). region influenced by quantum behavior. The hump in the specific-heat function, whose height is damped by the quantum fluctuations, enters the quantum region (see Fig. 6, $\gamma$ $=2$ ). Further increase of quantum fluctuations wipes out the hump, $\gamma=1$ in Fig. 6.

The systems of large asymmetry become sensitive to quantum effects for small values of $\gamma$, when the higher peak, corresponding to level crossing, enters the quantum range; quantum modification of the on-site potential reduces the height of the peak (Fig. 7).

The above analysis confirms a prediction that one parameter, $\gamma$, governs the quantum fluctuations in the system.

\section{B. Effects of discreteness: $k \leqslant 1$}

Discrete effects are becoming essential in weakly coupled systems, $k<1$. Their actual influence appears to be quite important in the dynamics, where localized excitations, unstable in continuum limit bell shapes, are stabilized due to discreteness [22]. Other significant effects are related to the

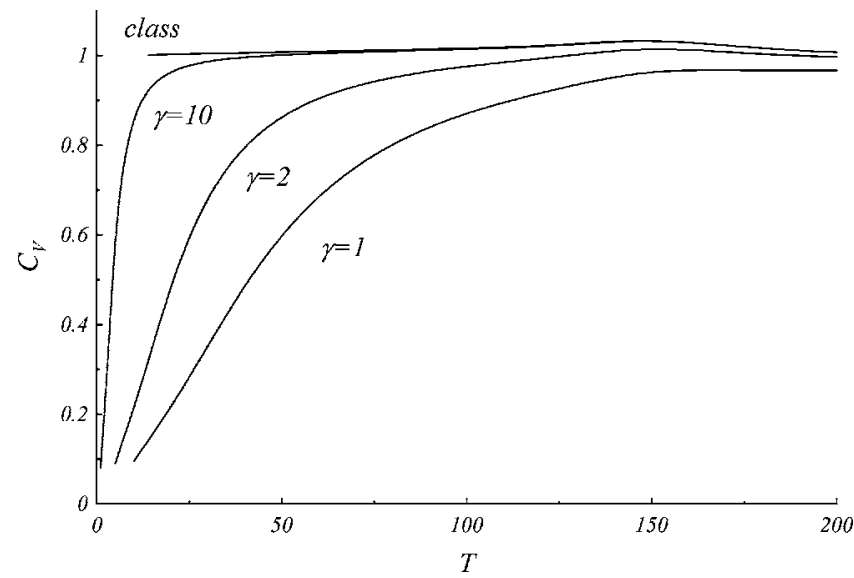

FIG. 6. Quantum effects in specific heat (small asymmetry: $V_{1}$ $\left.=0.4, V_{2}=0.5, \alpha_{1}=0.8, \alpha_{2}=0.8, q_{0}=7, k=100\right)$ for various values of quantum parameter $\gamma$. emergence of breathers. A naive expectation was that stabilization of bell shape excitations would be reflected in the thermodynamics: it means that discrete systems with an asymmetric double-well potential should reveal more similar behavior to the systems with a symmetric double-well potential than their continuous counterpartners. However, even in the continuum limit the thermodynamics of systems with symmetric and asymmetric on-site potential were found to be similar to each other for small asymmetry despite the differences in their dynamic properties.

In the context of our discussion of the thermodynamic properties of the 1D model, discreteness is taken into account (in the classical limit) modifying the method of evaluation of the free energy. In the continuum limit, thermodynamics of that $1 \mathrm{D}$ problem is described by the ground state of the "zero-dimensional problem," fictitious particle of mass $m^{*} \equiv \beta^{2} k \gamma^{2}$ proportional to interaction constant $k$ living within an on-site potential, perfectly satisfying a dimensional crossover hypothesis. In the discrete case, a question of finding a free energy is still expressed as an eigenvalue problem for an appropriate integral (not differential) operator. One can expect that for diminishing values of coupling parameter $k$, the differential Schrödinger-type equation becomes less applicable; instead, the integral eigenvalue approach (14) and (15) should be applied. Therefore, the thermodynamic properties are studied by means of the ground eigenvalue and corresponding ground eigenstate of the integral eigenvalue problem (14),

$$
\int K(x, y) \Psi_{n}(y) d y=\varepsilon_{n} \Psi_{n}(x),
$$

where an integral operator $K$ is explicitly given by Eq. (15).

There are a limited number of tools that enable a qualitative discussion of solutions of the above eigenvalue problem. We invoked here numerical methods: problem (14) has been solved within and beyond the classical limit. The results are presented for selected model parameters in Fig. 8: though the RTCT method and the following interpretation could not be applied here, the general conclusions are preserved. One can 


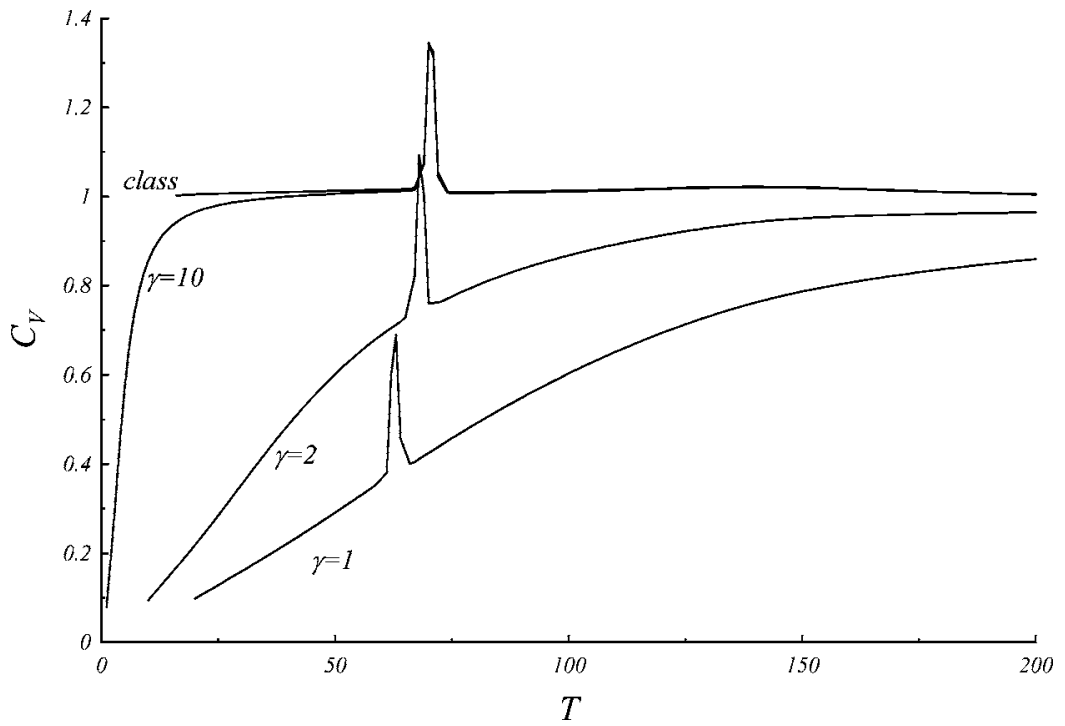

FIG. 7. Quantum effects in specific heat (large asymmetry: $V_{1}=0.4, V_{2}=0.5, \alpha_{1}=0.7, \alpha_{2}=1, q_{0}$ $=7, k=100)$ for various values of quantum parameter $\gamma$.

observe double-peak structure of specific heat corresponding to the avoided-level crossing. As one can see, discreteness suppresses intensity of peaks in specific heat. So, in a sense, it acts in a way similar to quantum fluctuations. The regime where discreteness turns out to be a key factor governing the overall picture is a large asymmetry limit. In fact, the high and narrow peak in specific heat of the continuum limit, manifesting in a coherent "jump" of the whole system between the pure states, should be strongly suppressed; such a "jump" should disappear in the limit of independent lattice sites $(k=0)$. As can be seen in Fig. 8, growing discreteness (diminishing $k$ ) leads to that behavior: the narrow peak in specific heat turns out to be suppressed in the discrete system $(k \ll 1)$, but it is still present, though shifted, to a very low value of $k$.

\section{SYSTEMS WITH ASYMMETRIC, TRIPLE-WELL POTENTIAL}

In our example of a triple-well potential, side wells are symmetric and a central well may be shallower or deeper.
The properties of that class of systems are studied by means of methods applied above for double-well potential systems. The discussion here will be limited to the case of the classical continuum approximation: quantum and discrete effects result in foreseeable changes. Depending on the interplay between depth and width of the middle and side wells for that triple-well potential, a one-or two-peak structure of the specific-heat function may also be observed. A smooth hump, associated with the appearance of large-amplitude excitations, appears in the range of high enough temperatures where, apart from small-amplitude oscillations, largeamplitude, localized excitations occur. That process is accompanied by leaking of the PDF through a region of the barrier. On the basis of the analysis in Sec. IV, one can make a prediction of when another, higher peak may be developed in the case of systems with a triple-well potential. That will happen due to the intersection of the unperturbed, single-well ground energy levels $E_{i}^{0}$ at some specific temperature. Provided that this temperature is lower than the temperature when $\left\{\max E_{i}^{0}\right\}$ reaches the edge of an energy barrier, the second, sharp peak will occur in the specific-heat function.
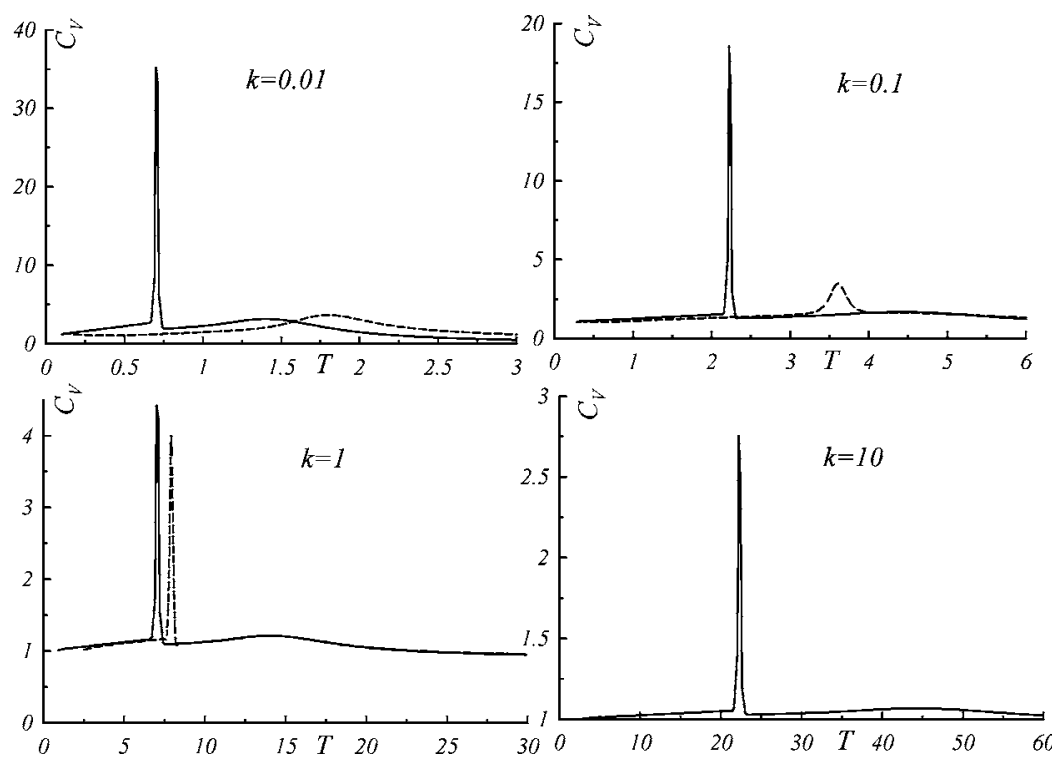

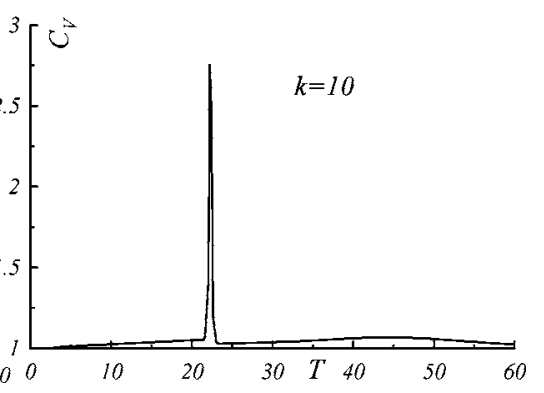

FIG. 8. Discrete effects in specific heat (large asymmetry: $V_{1}=0.4, V_{2}=0.5, \alpha_{1}=0.7, \alpha_{2}=1, q_{0}$ $=7, \gamma=10)$ for various values of parameter $k$ : continuum approximation (solid lines) and discrete results (dashed lines). 


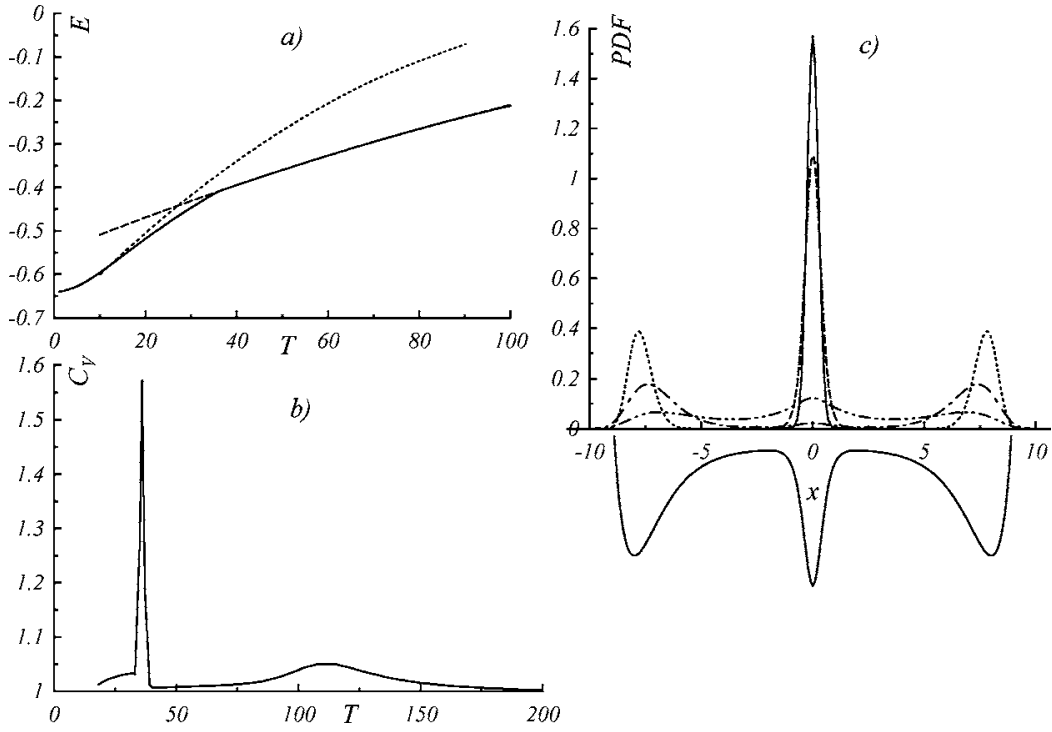

FIG. 9. Triple-well potential for values of parameters: $V_{1}=V_{2}=0.55, \alpha_{1}=\alpha_{2}=0.8, q_{0}=8, V_{0}$ $=0.7, \alpha=0.55, p_{0}=0, k=100, \gamma=10$. (a) Triplewell ground energy level (solid line) and singlewell energy levels corresponding to side and middle well (dashed and dotted lines), obtained via zeroth-order RTCT; (b) specific-heat function; (c) PDF for a few chosen temperatures: $T=20$ (solid), $T=35$ (dash), $T=37$ (dot), $T=100$ (dashdot), $T=140$ (dash-dot-dot).
To fulfill this condition, the energy-level crossing - as in the case of the double-well potential's "large" asymmetry (the deeper well being both side or middle in that case)—should also be narrower. For numerical reasons, the following form of the triple-well potential has been chosen:

$$
V_{3}(x)=V_{\mathrm{DM}}(x)+V_{\mathrm{PT}}(x),
$$

where $V_{\mathrm{DM}}(x)$ is the double-Morse potential (2) and $V_{\mathrm{PT}}(x)$ is the Pöschl-Teller potential,

$$
V_{\mathrm{PT}}(x)=-\frac{V_{0}}{\cosh ^{2}\left[\frac{x-p_{0}}{\alpha}\right]} .
$$

In Figs. 9(a) and 10(a), two examples of such potentials are shown; the energy of the true ground state of the system, $E^{0}$, and pure ground states in the wells, $E_{i}^{0}$, is indicated. In Figs. 9(b) and 10(b), the PDF for these two cases is presented, and in Figs. 9(c) and 10(c), the corresponding specific-heat functions are presented.

\section{DISCUSSION}

The aim of this paper was to give a thorough discussion of thermodynamic properties of the lattice, one-dimensional systems with an asymmetric potential revealing entropydriven phase transformations. One of the motivations was to determine the thermodynamic context of such a minimal lattice model of photoinduced phase transitions, where the ground electronic state is a multiple-well potential. We have discussed the properties of an asymmetric double-well potential and a triple-well potential with symmetric side wells. The former of these two types of potential relates to a Koshino and Ogawa model [9] and a model of spin conversion [10]; the latter one relates to a generalization of three localized electronic states. Applying the Feynman-Kleinert approach to that class of systems, one finds a partition function in a semiclassical form with an effective temperature-dependent potential. Therefore, thermodynamic properties both in classical and in quantum regimes are studied by means of an appropriate integral or differential (pseudo-Schrödinger) ground-state eigenvalue problem. The semiclassical WKB-

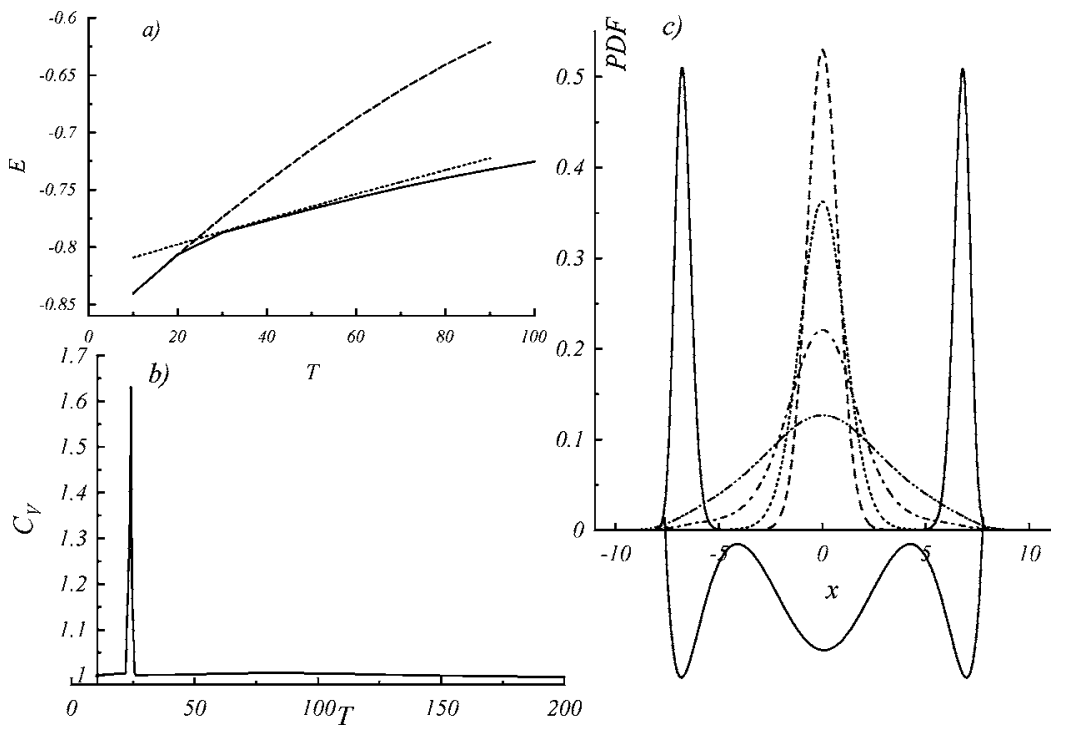

FIG. 10. Triple-well potential for values of parameters: $V_{1}=V_{2}=0.7, \alpha_{1}=\alpha_{2}=0.7, q_{0}=7, V_{0}$ $=0.8, \alpha=5, p_{0}=0, k=100, \gamma=10$. (a) Triple-well ground energy level (solid line) and single-well energy levels corresponding to side and middle well (dashed lines), obtained via zeroth-order RTCT; (b) specific-heat function; (c) PDF for a few chosen temperatures: $T=20$ (solid), $T=25$ (dash), $T=50$ (dot), $T=100$ (dash-dot), $T=250$ (dash-dot-dot). 
type approximation, namely the RTCT method applied for the pseudo-Schrödinger equation, provided the tools for a qualitative discussion and interpretation: in the case of a double-well potential, the true, double-well ground state may be regarded as a "mixture" of unperturbed, single-well ground states of left and right wells. Ground-state energy is interpreted as appropriate free energy: pure ground energy levels of left and right wells correspond to free energies associated with small oscillations (phonons) in left and right wells. Asymmetry of an on-site potential might be quantified as a "small" asymmetry, of "shift" type, and a "large" asymmetry, of "shape" type. The distinction between large and small asymmetry originates from temperature evolution of single-well ground states: they may (large asymmetry) or may not (small asymmetry) cross each other. Thermal evolution of a single-well state in a narrower well is faster than evolution in a broader well. Therefore, when the lower well is narrower than the shallower one, the single-well states may cross each other. Such a crossing, in this case described analytically, has to take place before the higher of these states reaches the edge of the potential barrier. It should be emphasized that the specific feature of our choice of doublewell potential is composed of two Morse potentials. Due to the exact solvability of a Morse potential, temperature evolution of single-well ground states (free energy of single-well phases) is described analytically. In the more general case of an arbitrary asymmetric double-well potential, the numerical rather than the analytical method is applied. Namely, evolution of the single-well states [Eq. (B2)],

$$
\cos \left[W_{i}\left(E_{i}^{n}\right)\right]=0,
$$

is found numerically for each of the wells separately. In the vicinity of their intersection, the true ground state is still given as a simple calculus problem, Eq. (B3). Asymmetry of an on-site potential is a key feature influencing temperature behavior of (ground) levels within the wells. As was formerly noticed [21], shift asymmetry is accompanied by a hump in the specific heat. Shape asymmetry may be accompanied, under particular circumstances, by a nearly singular, exponentially large peak in the specific-heat function, a hump being present in that case as well. The sharp peak is a result of an intersection of single-well ground energy levels, and the PDF of a system exhibits a dramatic "jump" between wells. It turns out that specific heat remains finite, as analytic evaluation confirms-there is no phase transition here, as one can expect in 1D system. Level crossing in systems with a large energy barrier between distinct (pure) phases at the crossing temperature should also be accompanied by other interesting effects. The results of preliminary Monte Carlo simulations [23] for thermodynamics display the mutual intersection of pure phases in the vicinity of the crossing point (see Fig. 11). In the case of a system of large asymmetry, studying equilibrium configurations one can observe the formation of "bubbles" or strings: finite length regions of a less stable phase are incorporated into a more stable phase. That observation is made in the vicinity of the crossing/ transformation point. These static properties are reflected in the dynamics of the system (studied at particular temperatures by means of a Nose method, following the Peyrard et
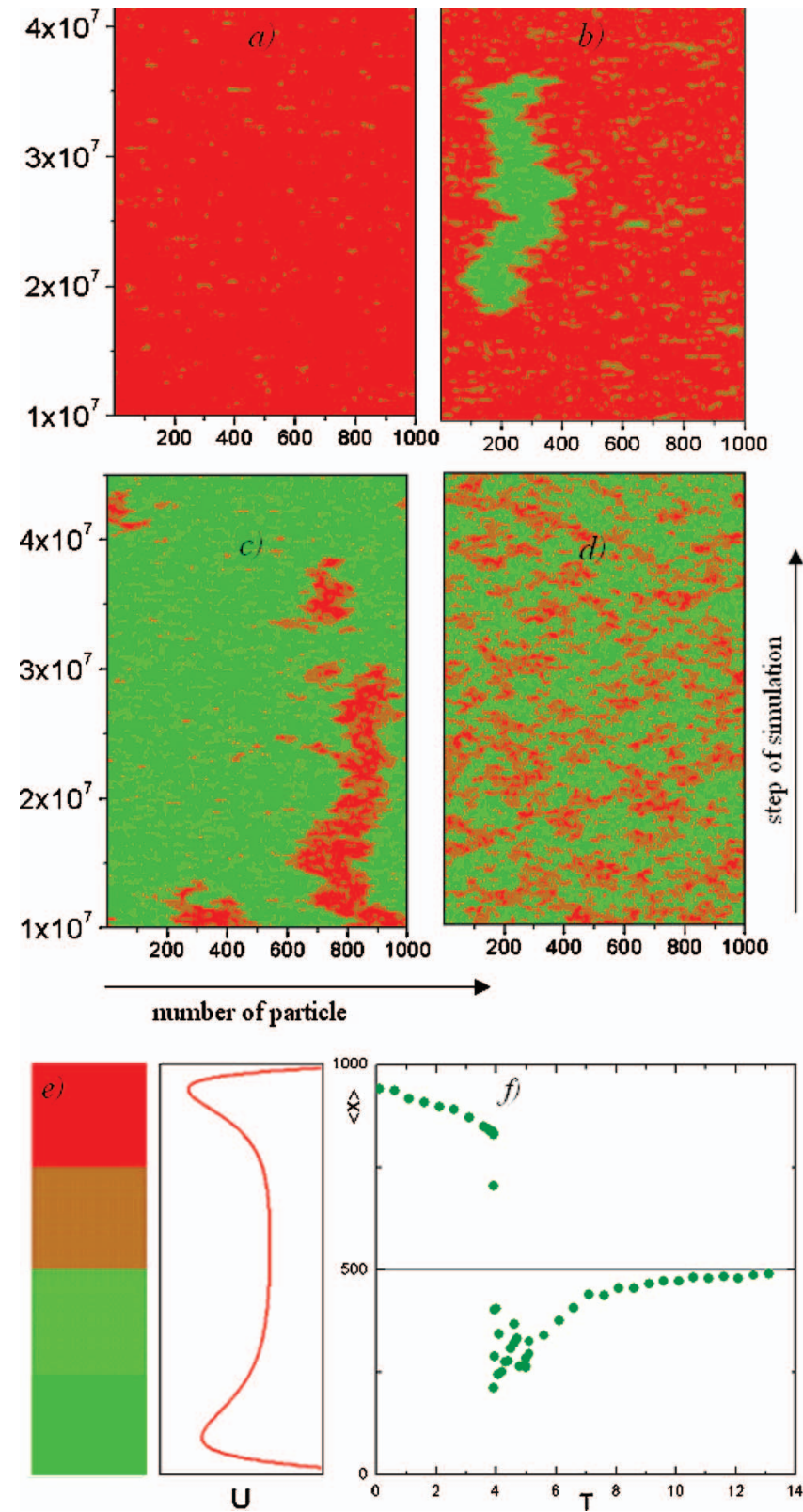

FIG. 11. (Color) Equilibrium configurations of particles for model parameters: $V_{1}=0.5, V_{2}=0.6, \alpha_{1}=0.7, \alpha_{2}=0.95, q_{0}=7, k$ =10, $\gamma=1$ for temperatures (a) $T=3$, (b) $T=3.8$, (c) $T=4.99$, (d) $T$ $=10$, (e) map of colors used, (f) average position vs temperature.

al. [24] application): bell-shape, localized excitations are observed in the neighborhood of the phase transformation point (see Fig. 12). The coexistence of finite length strings of an unstable phase incorporated into a stable phase is a characteristic for that type (large asymmetry) of system. It appears to be an interesting aspect of investigated systems, especially in view of experimentally observed finite length "strings" in photoinduced phase transitions; that problem will be the subject of a separate discussion. It should be added that large asymmetry, as related to crossing single-well ground levels, does not necessarily lead in 1D systems to a nearly singular manifestation. In fact, such a dramatic manifestation (in specific heat and PDF) is attributed to the situation of distinct phases with an exponentially large energy barrier between 

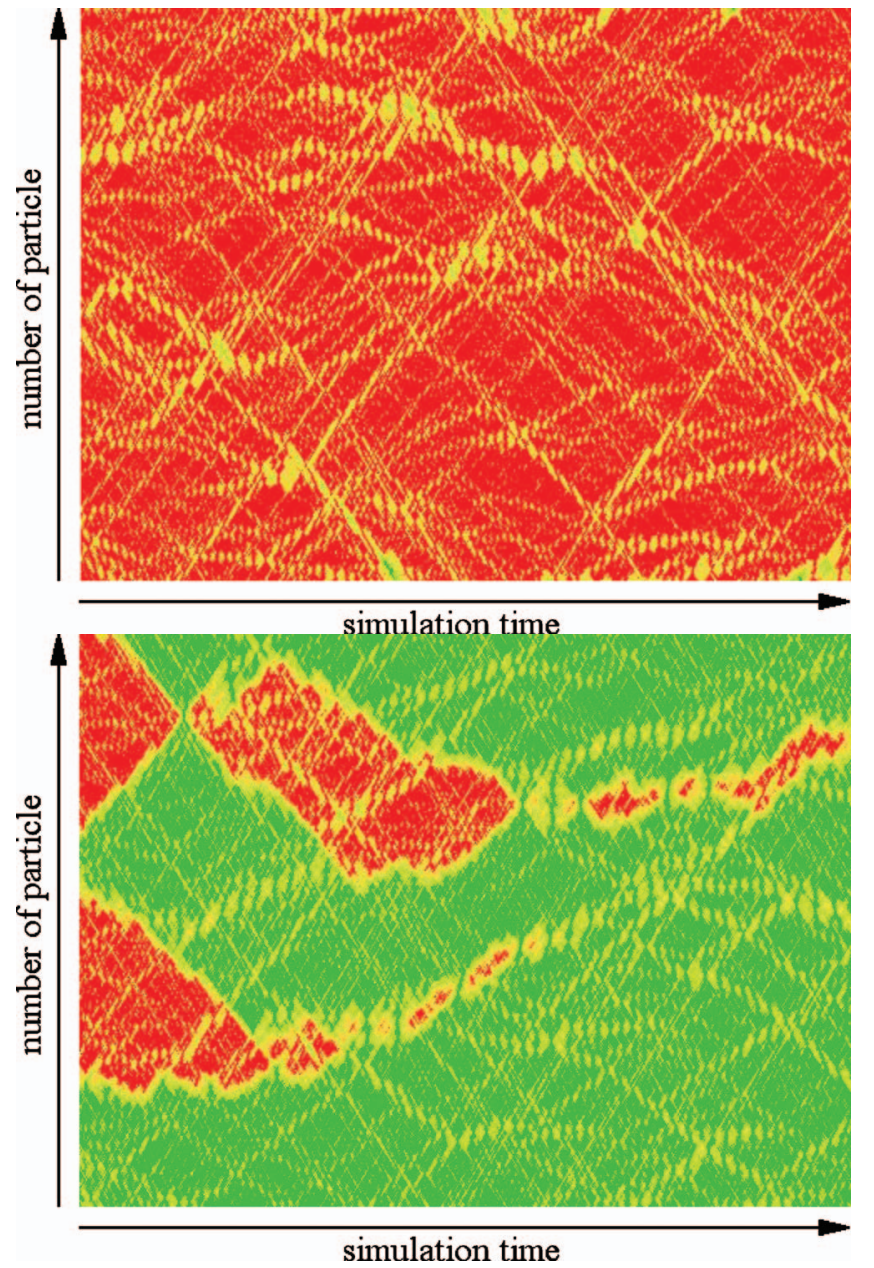

FIG. 12. (Color) Dynamics of particles for model parameters: $V_{1}=0.5, V_{2}=0.6, \alpha_{1}=0.7, \alpha_{2}=0.95, q_{0}=7, k=10$, and $\gamma=1$ for temperature (a) $T=3.8$, (b) $T=4.4$.

them. When the crossing takes place for less pronounced, "softer" barriers, it may cause less dramatic effects in specific heat (see Fig. 13). It is even more interesting, then, to investigate the consequences of level crossing (large asymmetry systems) observed for 1D systems for the properties of these systems in higher dimensions.

In a quantum regime, an on-site potential is substituted by an effective one but the characteristic features of the abovedescribed behavior are preserved. Namely, pure free energies (single-well ground energies) of the subsystems (wells) are getting mixed when the temperature rises. Quantum effects might affect the scenario of the phase transformation in the following way: the on-site potential becomes modified, the barrier becomes lowered, and the wells become shallower. Specific heat is modified in the range of low temperatures, and depending on the value of the parameter $\gamma$, it may, $\gamma$ $\approx 1$, or may not, $\gamma \gg 1$, affect entropy-driven phase transformation. The temperature dependence of the specific heat in the former case resembles the experimentally observed dependence for the spin crossover systems underlying the quantum character of these compounds-the quantum effects do not affect thermodynamics in an unexpected way.

Similarly to in the case of the symmetric on-site potential, discreteness suppresses the changes, e.g., diminishing the in- tensity and shifting peaks in the specific heat toward lower temperatures. Growing discreteness, regarded as weakening intersite coupling, is then studied by using the integral instead of the differential eigenvalue problem. Solving numerically that problem, one can find a quantitative rather than a qualitative difference between thermodynamics in the continuum limit and in a discrete case. Discreteness seems to be most important in the case of large asymmetry: weakening coupling affects the high and narrow peak, which is getting less intense and shifted toward the hump, being eventually swept out in a limit of independent sites. The changes caused by the discreteness are rather smooth and foreseeable even in that limit.

Systems with an asymmetric triple-well potential (symmetric side wells) exhibit the structure of a specific heat similar to the one of the systems with a double-well potential. Two-peak structure accompanies large asymmetry systems, both in the case of deeper side wells and in the case of deeper central well. The high and narrow peak corresponds to the dramatic change of a PDF structure and to a background pseudo-quantum-mechanical mechanism, namely the intersection of single-well ground energy levels of side and central wells. The hump in specific heat reflects "leaking" of the PDF from a well pronounced, more compact structure to a less compact form. Such a two-peak form of specific heat is expected to be damped by quantum fluctuations in a quantum regime, i.e., when an energy barrier is comparable with the energy of quantum fluctuations. Discreteness also would lead here to foreseeable smoothening of the specific-heat function.

Summarizing, let us emphasize the following: the common feature of systems of "small asymmetry" and "large asymmetry" is a smooth hump in the specific heat. That hump, being regarded as a manifestation of kinks in the case of a symmetric double-well potential, turns out to be present in a wide class of systems displaying strong nonlinearity, of multiple-well type, either symmetric or asymmetric. Nearly singular behavior of specific heat appears to be a peculiar feature of systems of "large" asymmetry. A sharp (but always finite) peak may be influenced by discrete effects; quantum effects turn out to be less influential. The interesting issue relates to the physical meaning of the phase transformations in systems of higher dimensions. In the case of one dimensional systems, "small" asymmetry is associated with one phase transformation and "large" asymmetry corresponds to two phase transformations. Due to their origin, these phase transformations should be referred to as entropy-driven phase transformations; a spectacular sequence of two following phase transformations is most clearly visible in an averaged position (Fig. 4) revealing behavior unique for large, shape asymmetry. Are entropy-driven phase transformations in one dimension going to convert into entropydriven phase transitions (of first order) in higher dimensions? That question seems to be intriguing also for other reasons. Recently, Boukhedadden [10] reported the possibility of a 1D low-spin-high-spin transformation in a spin-conversion system exhibiting a sharp peak in specific heat. The sharpness of that peak was, however, the result of anharmonicity of intersite coupling rather than asymmetry of an on-site potential. As argued by Morris and Gooding [7], the sharpness 

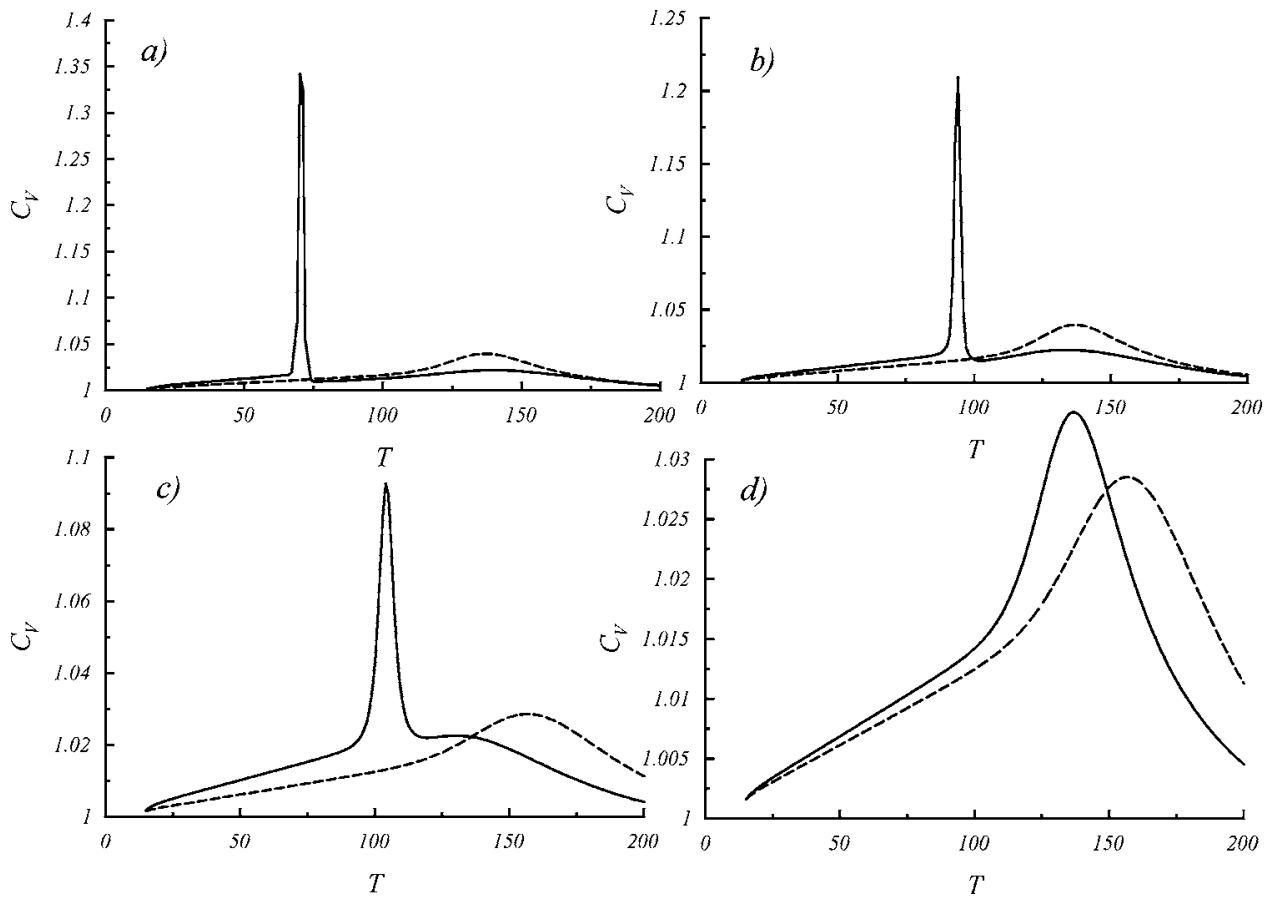
d)

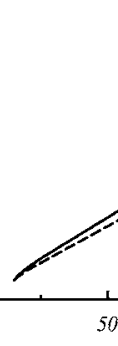

of the entropy change in the first-order phase transition in two-dimensional systems with a triple-well on-site potential is actually caused by the anharmonic interaction term. Is such an anharmonicity of interaction an alternative to a large asymmetry of the on-site potential mechanism for the sharp peak of the specific-heat 1D system? Or, in other words, are there two alternatives in 1D systems-large asymmetry of the on-site potential and anharmonicity of an intersite term-as precursors of a phase transition of the first order in higher dimensions? One can rather expect that the driving mechanism leading to first-order phase transition in higher dimensions would be crossing of "single-well" ground states corresponding to "pure" phases-it should correspond then to the systems of large asymmetry. That problem, apart from the further discussion of thermodynamics of photoinduced phase transitions and the possibilities of other types of lattice-driven phase transformation, will be considered in future papers.

\section{ACKNOWLEDGMENTS}

This work was carried out in part under British Council Grant No. WAR/341/244. A.R. wishes also to acknowledge the project PBZ-MIN-008/P03/2003 for the support.

\section{APPENDIX A}

The quantum-mechanical partition function may be expressed as a functional integral,

$$
\begin{aligned}
Z=\operatorname{Tr} e^{-\beta H} & =\int \prod_{n} d x_{n}^{\prime}\left\langle\bar{x}^{\prime}\left|e^{-\beta H}\right| \bar{x}^{\prime}\right\rangle \\
& =\int \prod_{n} D\left[x_{n}(\tau)\right] \exp \left(-\frac{1}{\hbar} A[\bar{x}(\tau)]\right),
\end{aligned}
$$

where an action $A$ is an integral over imaginary time,
FIG. 13. Large asymmetry (solid lines) evolving toward small asymmetry (dashed lines). Small asymmetry potential parameters: $V_{1}=0.4, V_{2}=0.5, \alpha_{1}=0.7$, $\alpha_{2}=0.7, \quad q_{0}=7, \quad k=100, \quad \gamma=10$; large asymmetry potentials parameters: $V_{1}=0.4, \quad V_{2}=0.5, \quad \alpha_{1}$ $=0.7, q_{0}=7, k=100, \gamma=10 . \alpha_{2}=1$ (a); 0.95 (b); 0.9 (c); 0.8 (d).

$$
\begin{gathered}
A[\bar{x}(\tau)]=\int_{0}^{T} \sum_{n}\left[\frac{M}{2} \dot{x}_{n}^{2}+V\left[x_{n}(\tau)\right]+\frac{1}{2} k\left[x_{n}(\tau)-x_{n+1}(\tau)\right]^{2}\right] d \tau, \\
T=\hbar \beta .
\end{gathered}
$$

Expanding trajectories in imaginary time around mean values,

$$
x_{n}(\tau)=x_{n}^{0}+\sum_{m=1}^{\infty}\left[x_{n}^{m} e^{-i \omega_{m} \tau}+x_{n}^{m^{*}} e^{i \omega_{m} \tau}\right],
$$

where $\omega_{m}=2 \pi m / \hbar \beta$ are Matsubara frequencies, one gets

$$
\begin{aligned}
& Z=\int \prod_{n} \frac{d x_{n}^{0}}{\sqrt{\frac{2 \pi \hbar^{2}}{M k_{B} T}}} \prod_{m=1}^{\infty}\left[\int \frac{d x_{n}^{m} \operatorname{Re} d x_{n}^{m} \operatorname{Im}}{\frac{\pi k_{B} T}{M \omega_{m}^{2}}}\right] \exp \left\{-\frac{M}{k_{B} T}\right. \\
& \times \sum_{n} \sum_{m=1}^{\infty} \omega_{m}^{2}\left|x_{n}^{m}\right|^{2}-\frac{1}{\hbar} \int_{0}^{\hbar \beta} d \tau\left[V \left(\left\{x_{n}^{0}+\sum_{m=1}^{\infty}\left[x_{n}^{m} e^{-i \omega_{m} \tau}\right.\right.\right.\right. \\
& \left.\left.\left.+x_{n}^{m^{*}} e^{i \omega_{m} \tau}\right]\right\}\right)-\frac{1}{\hbar} \int_{0}^{\hbar \beta} d \tau \sum_{n} \frac{1}{2} k\left[x_{n}^{0}+\sum_{m=1}^{\infty}\left[x_{n}^{m} e^{-i \omega_{m} \tau}\right.\right. \\
& \left.\left.\left.+x_{n}^{m^{*}} e^{i \omega_{m} \tau}\right]-x_{n-1}^{0}-\sum_{m=1}^{\infty}\left[x_{n-1}^{m} e^{-i \omega_{m} \tau}+x_{n-1}^{m^{*}} e^{i \omega_{m} \tau}\right]\right]^{2}\right\} \\
& \equiv \int \frac{d x_{n}^{0^{N}}}{\left[\frac{2 \pi \hbar^{2} \beta}{M}\right]^{N / 2}} \exp \left\{-\beta V_{\text {eff }}\right\} .
\end{aligned}
$$

Let $\hat{U}$ be a unitary matrix that diagonalizes the harmonic part of Hamiltonian $\hat{H}, \bar{u}=\hat{U} \bar{x}$, 


$$
\begin{aligned}
H=\sum_{q}\left[\frac{1}{2} M \dot{u}_{q}^{2}+\frac{1}{2} M \omega_{q}^{2} u_{q}^{2}\right]+V_{\mathrm{anh}}\left(\left\{\sum_{q} U_{n q} u_{q}\right\}\right), \\
u_{q}= \begin{cases}\operatorname{Re}\left[\sqrt{\frac{2}{N}} \sum_{n} e^{i q n} x_{n}\right], & q<0 \\
\operatorname{Im}\left[\sqrt{\frac{2}{N}} \sum_{n} e^{i q n} x_{n}\right], & q>0\end{cases}
\end{aligned}
$$

In these new variables, the action reads

$$
\begin{aligned}
A= & \sum_{q} \int_{0}^{\hbar \beta} d \tau\left[\frac{1}{2} M \dot{u}_{q}^{2}+\frac{1}{2} M \omega_{q}^{2} u_{q}^{2}\right] \\
& +\int_{0}^{\hbar \beta} d \tau V_{\mathrm{anh}}\left(\left\{\sum_{q} U_{n q} u_{q}(\tau)\right\}\right) .
\end{aligned}
$$

We define a local trial action as

$$
A_{\tilde{\tilde{\Omega}}}^{\left\{x_{0}\right\}}=\sum_{n} \int_{0}^{\hbar \beta} d \tau\left[\frac{1}{2} M \dot{x}_{n}^{2}+\frac{1}{2} M \widetilde{\Omega}_{m n}^{2}\left(x_{m}-x_{m}^{0}\right)\left(x_{m}-x_{m}^{0}\right)\right]
$$

with the matrix of trial frequencies $\hat{\widetilde{\Omega}}^{2}$ chosen so as to be diagonalized by matrix $\hat{U}$,

$$
A_{\tilde{\omega}}^{\left\{u_{0}\right\}}=\sum_{q} \int_{0}^{\hbar \beta} d \tau\left[\frac{1}{2} M \dot{u}_{q}^{2}+\frac{1}{2} M \widetilde{\omega}_{q}^{2}\left(u_{q}-u_{q}^{0}\right)^{2}\right] .
$$

As matrix $\hat{U}$ is unitary, $\int d u_{q}^{0^{N}}=\int d x_{q}^{0^{N}}$, and

$$
Z=e^{-F / k_{B} T}=\int \frac{d u_{q}^{0^{N}}}{\left[\frac{2 \pi \hbar^{2} \beta}{M}\right]^{N / 2}} \exp \left\{-\beta V^{\mathrm{eff}}\right\}
$$

with

$$
e^{-V^{\mathrm{eff} / k_{B} T}}=Z_{0}\left\langle\exp \left\{-\frac{1}{\hbar}\left[A-A_{\widetilde{\omega_{q}}}^{u_{0}}\right]\right\}\right) \underset{\left\{\tilde{\omega_{q}}\right\}}{\left\{u_{0}\right\}},
$$

where the averaging operator denoted by $\langle\cdots\rangle_{\left\{\tilde{\omega}_{q}\right\}}^{\left\{u_{0}\right\}}$ is defined as

$$
\langle F\rangle_{\omega_{q}}^{u_{0}}=\left[Z_{0}\right]^{-1} \prod_{q} \prod_{m=1}^{\infty}\left[\int \frac{d u_{q}^{m \operatorname{Re}} d u_{q}^{m \operatorname{Im}}}{\frac{\pi k_{B} T}{M \omega_{m}^{2}}}\right] F e^{(1 / \hbar) A_{\left\{\omega_{q}\right\}}^{\left\{u_{0}\right\}}}
$$

and

$$
Z_{0}=\prod_{q} \prod_{m=1}^{\infty}\left[\int \frac{d u_{q}^{m \operatorname{Re}} d u_{q}^{m \operatorname{Im}}}{\frac{\pi k_{B} T}{M \omega_{m}^{2}}}\right] e^{(1 / \hbar))_{\omega_{q}}^{u_{0}}} .
$$

Using the Jensen-Peierls inequality,

$$
\left\langle\exp \left\{-\frac{1}{\hbar}\left[A-A_{\left\{\tilde{\omega}_{q}\right\}}^{\left\{u_{0}\right\}}\right]\right\}\right\rangle_{\left\{\tilde{\omega}_{q}\right\}}^{\left\{u_{0}\right\}} \geqslant \exp \left\{-\left\langle\frac{1}{\hbar}\left[A-A_{\left\{\tilde{\omega}_{q}\right\}}^{\left\{u_{0}\right\}}\right]\right\rangle_{\left\{\tilde{\omega}_{q}\right\}}^{\left\{u_{0}\right\}}\right\} .
$$

An estimation for effective potential is

$$
V^{\mathrm{eff}} \leqslant-k_{B} T \ln Z_{0}+k_{B} T\left\langle\frac{1}{\hbar} A-\frac{1}{\hbar} A_{\left\{\tilde{\omega}_{q}\right\}}^{\left\{u_{0}\right\}}\right\rangle_{\left\{\tilde{\omega}_{q}\right\}}^{\left\{u_{0}\right\}} \equiv \tilde{V}^{\text {eff }} .
$$

After some algebra, it turns out that the effective potential reads

$V^{\mathrm{eff}} \leqslant \sum_{n} \frac{1}{2} k\left(x_{n}-x_{n-1}\right)^{2}+\frac{1}{2} M \sum q\left[\left(\omega_{q}^{2}-\widetilde{\omega}_{q}^{2}\right) a_{q}^{2}\right]+F_{0}+V_{\mathrm{anh}}^{\mathrm{eff}}$,

where

$$
\omega_{q}^{2}=4 k \sin ^{2}\left[\frac{\pi}{N} q\right]
$$

$$
F_{0}=-k_{B} T \sum_{q} \ln \left[\frac{\frac{\hbar \widetilde{\omega}_{q}}{2 k_{B} T}}{\sinh \left(\frac{\hbar \widetilde{\omega}_{q}}{2 k_{B} T}\right)}\right] \text {, }
$$

$$
a_{q}^{2}=\frac{k_{B} T}{M \widetilde{\omega}_{q}^{2}}\left[\frac{\hbar \widetilde{\omega}_{q}}{2 k_{B} T} \operatorname{coth} \frac{\hbar \widetilde{\omega}_{q}}{2 k_{B} T}-1\right],
$$

$$
V_{\mathrm{anh}}^{\mathrm{eff}}=\sum_{n} \int \frac{d y_{n}}{\sqrt{2 \pi D}} \exp \left\{-\frac{1}{2} \frac{\left(y_{n}-x_{n}^{0}\right)^{2}}{a_{q}^{2} U_{n q}^{2}}\right\} V\left(y_{n}\right)
$$

with

$$
D \equiv \frac{1}{N} \sum_{q} a_{q}^{2} .
$$

One gets trial frequencies from a minimization condition,

$$
\begin{aligned}
\frac{d V^{\mathrm{eff}}}{d \widetilde{\omega}_{q}^{2}}=0 \Rightarrow \omega_{q}^{2}-\widetilde{\omega}_{q}^{2} & =\frac{2}{M} \frac{\partial V_{\mathrm{anh}}^{\mathrm{eff}}}{\partial a_{q}^{2}} \Rightarrow \widetilde{\omega}_{q}^{2}\left(\left\{x_{n}\right\}\right) \\
& =4 k \sin ^{2}\left[\frac{\pi}{N} q\right]-\frac{2}{M} \frac{\partial V_{\mathrm{anh}}^{\mathrm{eff}}\left(\left\{x_{n}\right\}\right)}{\partial a_{q}^{2}} .
\end{aligned}
$$

In the high-temperature limit, quantum parameters $a_{q}^{2}$, tend to zero and the effective potential regains its classical shape: $\lim _{T \rightarrow \infty} V^{\text {eff }}=V$.

In general, the matrix of trial frequencies $\hat{\widetilde{\Omega}}^{2}$ need not be chosen in such a way that will be diagonalized by the same 
matrix that diagonalizes the harmonic part of the Hamiltonian. However, applying the variational procedure-with respect to $a_{q}^{2}$ s - to the resulting effective potential gives the result that in the first-order approximation, the matrix $\hat{U}$ diagonalizes the matrix of trial frequencies [25]. We use this result from the very beginning. In the case of the doubleMorse potential,

$$
\begin{aligned}
V(y)= & V_{1}\left(e^{-2 \alpha_{1}\left(y+q_{0}\right)}-2 e^{-\alpha_{1}\left(y+q_{0}\right)}\right)+V_{2}\left(e^{2 \alpha_{2}\left(y-q_{0}\right)}\right. \\
& \left.-2 e^{\alpha_{2}\left(y-q_{0}\right)}\right),
\end{aligned}
$$

one gets a sum of integrals of Gaussian type, which may be solved by gathering to the quadratic form,

$$
\int \frac{d y_{n}}{\sqrt{2 \pi D}} \exp \left\{-\frac{1}{2} \frac{\left(y_{n}-x_{n}^{0}\right)^{2}}{D}-\alpha y_{n}\right\}=\exp \left\{-\alpha x_{n}+\frac{1}{2} \alpha^{2} D\right\}
$$

with $\alpha=\alpha_{1}, 2 \alpha_{1}, \alpha_{2}, 2 \alpha_{2}$ successively.

So

$$
\begin{aligned}
V_{\mathrm{DM}}^{\mathrm{eff}}= & \sum_{n}\left[V_{1}\left[e^{-2 \alpha_{1}\left(x_{n}+q_{0}\right)} e^{2 \alpha_{1}^{2} D}-2 e^{-\alpha_{1}\left(x_{n}+q_{0}\right)} e^{(1 / 2) \alpha_{1}^{2} D}\right]\right. \\
& \left.+V_{2}\left[e^{2 \alpha_{2}\left(x_{n}-q_{0}\right)} e^{2 \alpha_{2}^{2} D}-2 e^{\alpha_{2}\left(x_{n}-q_{0}\right)} e^{(1 / 2) \alpha_{2}^{2} D}\right]\right]
\end{aligned}
$$

self-consistently with Eqs. (A18) and (A20).

For numerical reasons, we will use here an approximation: $D$, which in general depends on coordinates $\left\{x_{n}\right\}$, will be taken invariant: $D\left(\left\{x_{n}\right\}\right) \equiv D\left(\left\{x_{\min }\right\}\right)$, where $x_{\min }$ means this value of coordinate $x_{n}$, constant along the chain, where the effective potential takes its minimum value: $V_{\mathrm{DM}}^{\text {eff }}$ $\left(\left\{x_{\min }\right\}\right)=0$. Also the limit of $N \rightarrow \infty$ will be taken for numerics.

\section{APPENDIX B}

The pseudo-Schrödinger eigenvalue problem for an asymmetric double well potential (2) might be solved by using the semiclassical, WKB-type approach, RTCT (real trajectories in complex time), developed in [20]. This method provides us with an explicit analytic formula determining energy levels (see Fig. 14),

$$
\cos \left[W_{1}(E)\right] \cos \left[W_{2}(E)\right]-\frac{1}{4} b^{2} \sin \left[W_{1}(E)\right] \sin \left[W_{2}(E)\right]=0,
$$

where

$$
\begin{gathered}
W_{i}(E)=\int_{b_{i-1}}^{a_{i}} d x \sqrt{2 m^{*}[E-V(x)]}, \\
b(E)=\exp \left[-\int_{a_{1}}^{b_{1}} d x \sqrt{2 m^{*}[V(x)-E]}\right]
\end{gathered}
$$

(see Fig. 14).

The ground-state eigenvalue $E_{0}$ of Eq. (B1) corresponds to the free energy of the original problem and evolves with the temperature via $m^{*} \propto T^{-2}$. To study the properties, we use a two-step approximation. A first step is to regard two wells

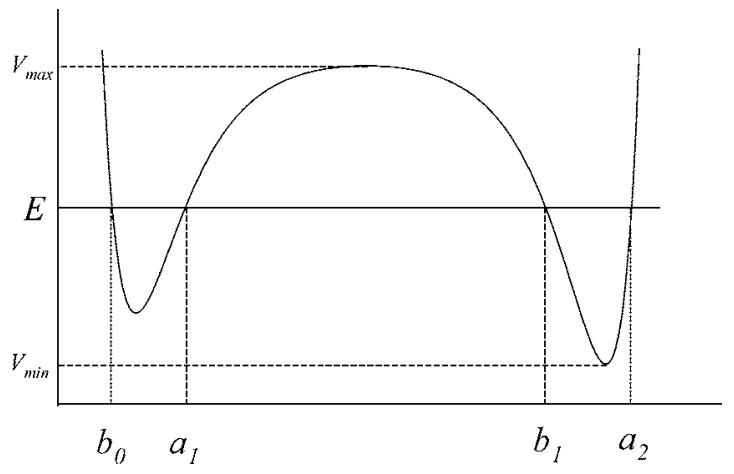

FIG. 14. Limits of integration in the RTCT method for the double-well potential.

of the potential as independent- this corresponds to the impenetrable, an opaque barrier, $b(E)=0$. Energy levels $E_{i}^{n}(i$ $=1,2)$ in the left $(1)$ and right (2) wells are then found as solutions (B1),

$$
\cos \left[W_{i}\left(E_{i}^{n}\right)\right]=0 .
$$

Single-well ground-state energy $E_{1}^{0}\left(E_{2}^{0}\right)$ corresponds to the ground state of particles of mass $m^{*}$ oscillating in the left (right) well. A second step is to take into account a finite value of the barrier parameter, $b(E) \neq 0$ : the actual, true, double-well ground-state energy is expressed as a mixture of "single-well" ground-state eigenvalues $E_{1}^{0}, E_{2}^{0}$. If $E_{1}^{0}$ and $E_{2}^{0}$ are close enough to each other, i.e., the distance between them is smaller than the spacing between ground and first excited levels within each of the wells, $\left|E_{1}^{0}-E_{2}^{0}\right|<\min \mid E_{i}^{1}$ $-E_{i}^{0} \mid$, then an analytic solution of Eq. (B1) might be found. A characteristic pair of solutions $E_{0}^{\mp}$ is given as

$$
\begin{gathered}
E_{0}^{\mp}=\frac{1}{2}\left[\left(E_{0}^{1}-E_{0}^{2}\right) \mp \sqrt{\left(E_{0}^{1}-E_{0}^{2}\right)^{2}+b^{2}\left(E_{0}\right) \nu_{1} \nu_{2}}\right], \\
\nu_{i}=\left[\left.\frac{\partial W_{i}}{\partial E}\right|_{E=E_{i}^{0}}\right]^{-1} .
\end{gathered}
$$

The temperature evolution of the "double-well" ground state $E_{0}^{-}$depends on the distance of "single-well" ground states $\left|E_{1}^{0}-E_{2}^{0}\right|$; that quantity itself depends on the arrangement and the shapes of both wells. In the case of double Morse potential (2), the temperature evolution of "singlewell" ground states is described analytically: for an arbitrary Morse potential,

$$
V_{M}(x)=V\left(e^{-2 \alpha\left(x+q_{0}\right)}-2 e^{-\alpha\left(x+q_{0}\right)}\right),
$$

the ground-state eigenvalue is known,

$$
E_{0}(T)=-\frac{T^{2}}{2} \frac{\alpha^{2}}{2 \gamma^{2}}\left[\frac{1}{2}-\sqrt{\frac{2 \gamma^{2} k V}{\alpha^{2} T^{2}}}\right]^{2} .
$$

In the case of a triple-well potential, energy levels are determined within RTCT by the following formula: 


$$
\begin{aligned}
\cos [ & \left.W_{1}(E)\right] \cos \left[W_{2}(E)\right] \cos \left[W_{3}(E)\right] \\
= & \frac{1}{4} b_{I I}^{2} \cos \left[W_{1}(E)\right] \sin \left[W_{2}(E)\right] \sin \left[W_{3}(E)\right] \\
& +\frac{1}{4} b_{I}^{2} \cos \left[W_{3}(E)\right] \sin \left[W_{1}(E)\right] \sin \left[W_{2}(E)\right] \\
& +\frac{1}{4} b_{I}^{2} \frac{1}{4} b_{I I}^{2} \cos \left[W_{2}(E)\right] \sin \left[W_{1}(E)\right] \sin \left[W_{3}(E)\right],
\end{aligned}
$$

where

$$
\begin{gathered}
W_{i}(E)=\int_{b_{i-1}}^{a_{i}} d x \sqrt{2 m^{*}[E-V(x)]}, \\
b_{i}(E)=\exp \left[-\int_{a_{i}}^{b_{i}} d x \sqrt{2 m^{*}[V(x)-E]}\right]
\end{gathered}
$$

(see Fig. 15).

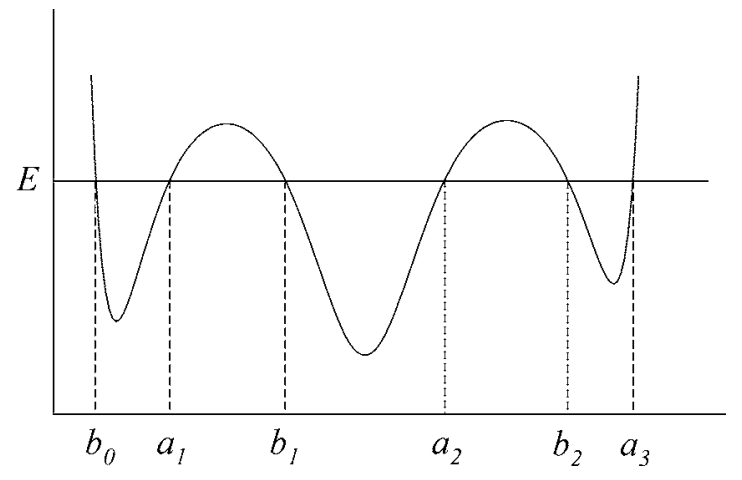

FIG. 15. Limits of integration in the RTCT method for the triple-well potential.

Single-well energy levels are defined by the first step, an opaque barrier approximation in the same way as above [Eq. (B2)]. Triple-well levels might be expressed via single-well levels in the case of close enough levels in a similar approximation as above, but in the paper only numerical results are used.
[1] V. Antonchenko, A. Davydov, and A. Zolotariuk, Phys. Status Solidi B 115, 631 (1983).

[2] A. Davydov, Solitons in Molecular Systems, 2nd ed. (Reidel, Dordrecht, 1990).

[3] M. Peyrard and A. R. Bishop, Phys. Rev. Lett. 62, 2755 (1989).

[4] F. J. Alexander, S. Habib, and A. Kovner, Phys. Rev. E 48, 4284 (1993).

[5] J. Currie, S. Trullinger, A. Bishop, and J. Krumhansl, Phys. Rev. B 15, 5567 (1997).

[6] S. Habib, A. Khare, and A. Saxena, Physica D 123, 341 (1998).

[7] J. R. Morris and R. J. Gooding, Phys. Rev. Lett. 65, 1769 (1990).

[8] K. Nasu, Rep. Prog. Phys. 67, 1607 (2004).

[9] K. Koshino and T. Ogawa, J. Phys. Soc. Jpn. 68, 2164 (1999).

[10] K. Boukheddaden, Prog. Theor. Phys. (to be published).

[11] K. Boukheddaden, I. Shteto, B. Hoo, and F. Varret, Phys. Rev. B 62, 14796 (2000); Phys. Rev. B 62, 14806, (2000).

[12] J. A. Nasser, K. Boukheddaden, and J. Linares, Eur. Phys. J. B 39, 219 (2004).

[13] K. Nasu, H. Ping, and H. Mizouchi, J. Phys.: Condens. Matter
13, R693 (2001).

[14] K. Yonemitsu and N. Miyashita, Phys. Rev. B 68, 075113 (2003).

[15] T. Luty, Acta Phys. Pol. A 87, 1009 (1995).

[16] P. Gütlich, A. Hauser, and H. Spiering, Angew. Chem., Int. Ed. Engl. 33, 2024 (1994).

[17] H. Kleinert, Path Integrals in Quantum Mechanics (World Scientific, Singapore, 2004).

[18] R. Giachetti and V. Tognetti, Phys. Rev. B 33, 7647 (1986).

[19] A. Khare, S. Habib, and A. Saxena, Phys. Rev. Lett. 79, 3797 (1997).

[20] A. Radosz and W. Magierski, J. Math. Phys. 33, 1745 (1992).

[21] A. Radosz, K. Ostasiewicz, P. Magnuszewski, and P. Machnikowski, Phys. Rev. E 64, 062103 (2001).

[22] P. Machnikowski, P. Magnuszewski, and A. Radosz, Phys. Rev. E 63, 016601 (2000).

[23] A. Radosz, K. Ostasiewicz, P. Magnuszewski, Ł. Radosiński, F. V. Kusmartsev, J. H. Samson, J. Damczyk, A. C. Mituś, and G. Pawlik (unpublished).

[24] T. Dauxois and M. Peyrard, Phys. Rev. E 47, 684 (1993).

[25] P. Machnikowski (unpublished). 